\title{
Coronal Magnetic Field Models
}

\author{
Thomas Wiegelmann' ${ }^{1}$ Gordon J.D. Petrie ${ }^{2}$ Pete Riley ${ }^{3}$
}

Received: 29 May 2015 / Accepted: 22 June 2015 / Published online: 31 July 2015

(C) The Author(s) 2015. This article is published with open access at Springerlink.com

\begin{abstract}
Coronal magnetic field models use photospheric field measurements as boundary condition to model the solar corona. We review in this paper the most common model assumptions, starting from MHD-models, magnetohydrostatics, force-free and finally potential field models. Each model in this list is somewhat less complex than the previous one and makes more restrictive assumptions by neglecting physical effects. The magnetohydrostatic approach neglects time-dependent phenomena and plasma flows, the force-free approach neglects additionally the gradient of the plasma pressure and the gravity force. This leads to the assumption of a vanishing Lorentz force and electric currents are parallel (or anti-parallel) to the magnetic field lines. Finally, the potential field approach neglects also these currents. We outline the main assumptions, benefits and limitations of these models both from a theoretical (how realistic are the models?) and a practical viewpoint (which computer resources to we need?). Finally we address the important problem of noisy and inconsistent photospheric boundary conditions and the possibility of using chromospheric and coronal observations to improve the models.
\end{abstract}

Keywords Sun · Corona · Magnetic Field · Active Region · MHD

\section{Why Do We Need to Model the Coronal Field?}

Because the most spectacular and dangerous manifestations of solar activity, flares and coronal mass ejections (CMEs), occur in the corona, and are almost certainly powered by free magnetic energy stored in the coronal magnetic field, modeling and measurement of the coronal field have been urgent goals of solar physics for many years. Only in the last decade

\footnotetext{
$凶$ T. Wiegelmann

wiegelmann@mps.mpg.de

1 Max-Planck-Institut für Sonnensystemforschung, Justus-von-Liebig-Weg 3, 37077 Göttingen, Germany

2 National Solar Observatory, 950 N. Cherry Ave., Tucson, AZ, 85719, USA

3 Predictive Science, 9990 Mesa Rim Road, Suite 170, San Diego, CA, USA
} 
Fig. 1 Contour map of the measured coronal magnetic field strength using Fe XIII overplotted on the EIT Fe XV image. The thick contour corresponds to $4 \mathrm{G}$, with additional contours corresponding to flux densities of 2, 0 , and $-2 \mathrm{G}$. Here the line-of-sight magnetic flux density and transverse field orientation were mapped in two dimensions with a sensitivity of about $1 \mathrm{G}$ and $20^{\prime \prime}$ spatial resolution after 70 minutes of integration. These measurements reveal the line-of-sight coronal magnetic field $100 \mathrm{Mm}$ above an active region to have a flux density of about $4 \mathrm{G}$. From Lin et al. (2004), Fig. 3. (C) AAS. Reproduced with permission

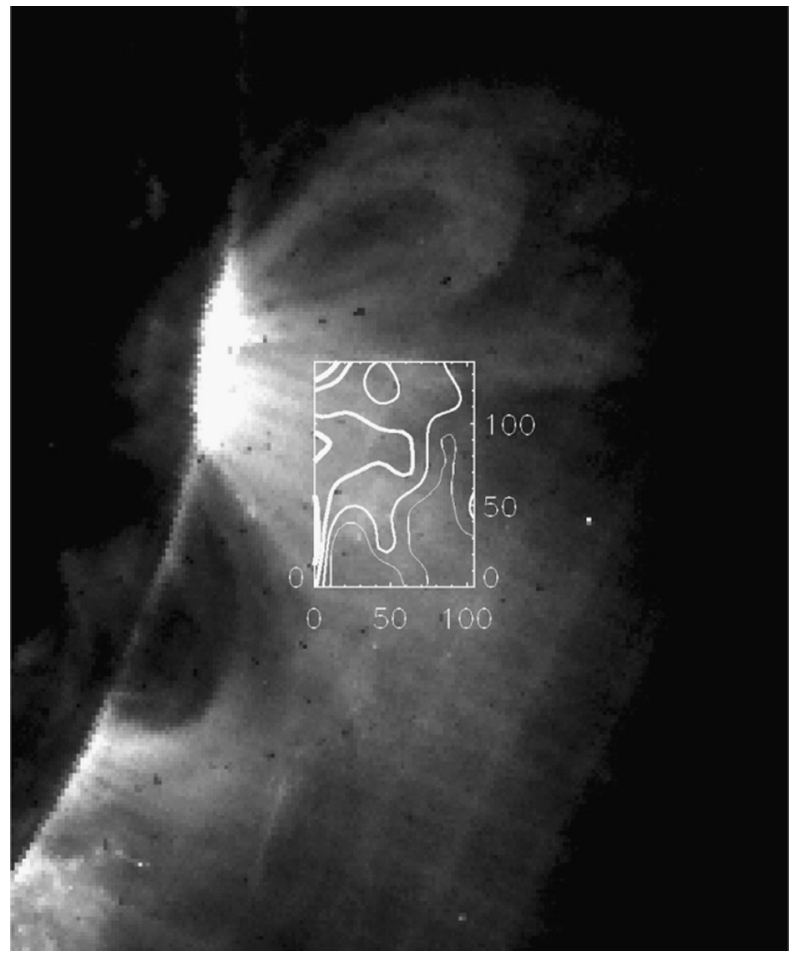

or so has Zeeman splitting of magnetically sensitive lines been successfully used to deduce the coronal magnetic flux density. Lin et al. (2004) mapped the line-of-sight flux density and transverse field orientation in two dimensions after a 70-minute integration with $1 \mathrm{G}$ sensitivity and $20^{\prime \prime}$ spatial resolution. Their coronal magnetogram is shown in Fig. 1, over-plotted on an SoHO/EIT EUV intensity image showing magnetic loop structure. The magnetogram indicates that the line-of-sight flux density of the loop system is decreasing with height, as is to be expected in a stratified atmosphere.

On the face of it, coronal magnetic field measurements might be expected to be quite straightforward. The structures are large and in plain sight. On the other hand, they are optically thin, making their geometrical interpretation difficult. Also the faintness of the signal in coronal spectro-polarimetric measurements makes it challenging to determine the coronal magnetic field. Also there are ambiguities in the transverse field measurements, specifically the $180^{\circ}$ ambiguity and the Van Vleck ambiguity (Rachmeler et al. 2013). The photospheric and chromospheric signals are much stronger and the field measurements easier to interpret. Regular measurements have been available from the photosphere since the 1960s and 1970s (Howard 1967; Livingston et al. 1976; Svalgaard et al. 1978), and from the chromosphere since the 1980s (Jones 1985). Quantitative information for the coronal magnetic field has mainly been provided by models extrapolated from more accessible measurements deriving from lower atmospheric layers, usually photospheric but sometimes chromospheric. These models are based on simplifying assumptions regarding both their governing equations and the treatment of the lower-boundary data. The basic assumptions are that surface magnetograms can be used to represent accurately the flux magnetic field distribution across the base of the corona, and the magnetic structure of the corona can then be effectively approximated by a simplified model extrapolated from these lower-boundary data. 
Some comparisons between extrapolated coronal field models and direct coronal field measurements have been carried out (Liu and Lin 2008). Usually, however, the structure of the coronal magnetic field is interpreted from, e.g., EUV intensity images as in Fig. 1. For example, the fibril loop structure seen in coronal images is often assumed to be aligned with the magnetic vector field. This assumption is based on the facts that the plasma is electrically highly conducting so that there is no plasma flow across field lines, the plasma is hot enough for the gradients along the loop to be much smaller than those across, and the thermal conduction is much more efficient along fields than across, keeping cross-field temperature gradients much higher than field-aligned temperature gradients.

In the following sections we will focus on the four main types of coronal model, the magnetohydrodynamics (MHD) model, the magnetohydrostatic (MHS) model, the nonlinear force-free field (NLFFF) model and the potential-field source-surface (PFSS) model, in decreasing order of sophistication. While these four models differ enormously in complexity, they all represent simplifying approximations of the real corona. Within this review we go from complex to simple models, compared to the usual direction of such reviews because we discuss more and more physical assumptions (instead of physical ingredients) as we proceed. The intention is to emphasize each new assumption's effects (strengths and limitations) at each step. We will describe the physical assumptions underlying these approximations, and we will illustrate the approximations with examples. We will proceed in descending order of complexity, beginning with MHD and ending with potential fields, describing at each step the additional assumptions behind each successive approximation.

\section{Magnetohydrodynamics in the Solar Corona}

MHD describes the interaction between a magnetized fluid, i.e., a plasma, and a magnetic field. Many descriptions of such interactions exist, offering different compromises between physical complexity and practical simplicity, from a full $N$-body problem to single-fluid MHD. Here we confine ourselves to single-fluid MHD, since this is the most widely used plasma physics approximation in coronal modeling, and the only self-consistent approach for modeling the interaction between the global coronal magnetic field and plasma that is viable with present-day computational resources.

The validity of the MHD approximation relies on the plasma behaving like a fluid. The usual condition for a fluid description is that the mean free path $\lambda_{c}$ is much smaller than the characteristic length scales of the system, $\lambda_{c} \ll \nabla f / f$ for all quantities $f$. But $\lambda_{c} \propto T^{2}$ where $T$ is the temperature, so this fluid condition may be violated for hot plasmas, e.g., the several-MK plasmas in the solar corona.

Fortunately, as far as the corona is concerned, this argument is misleading. At coronal temperatures, the particles are almost all ionized, and electrostatic interaction prevents large-scale separation of opposite charges, so the large-scale dynamics are controlled by the magnetic field $\mathbf{B}$.

In a uniform field $\mathbf{B}$, a charged particle moves helically along a field line with Larmor radius $r_{L}=v m / Z e B$ and with gyrofrequency $\Omega=Z e B / m$, where $B=|\mathbf{B}|, v$ is the speed of the particle in the plane of the circle associated with $r_{L}, m$ and $Z e$ are its mass and charge, and $e$ is the charge of an electron. This determines the length scale of the particle motion in the plane perpendicular to the field.

Along the field, the particle drifts freely until it collides with another particle. Coulomb collisions, near-encounters with other particles that scatter the particle from its helical 
path, occur at a frequency $v_{c}=1.3 \times 10^{-20} n /\left(m^{1 / 2} T^{3 / 2}\right)$ where $n$ is the particle number density. The drift distance between collisions is the mean free path $\lambda_{c}=v_{\mathrm{th}} / v_{c}$ where $v_{\text {th }}=(k B T / m)^{1 / 2}$ is the typical thermal speed of the particles.

In the corona, the Larmor radius $r_{L} \approx 1 \mathrm{~m}$ and $20 \mathrm{~mm}$, and the gyrofrequency $\Omega=$ $10^{5} \mathrm{rad} \mathrm{s}^{-1}$ and $10^{7} \mathrm{rads}^{-1}$ for ions and electrons, respectively. Collision frequencies are $0.1 \mathrm{~s}^{-1}$ and $5 \mathrm{~s}^{-1}$, giving a mean free path of order $1000 \mathrm{~km}$ along the field.

In cool loops and prominences the collision frequency is much higher, and the mean free path much smaller, because of the lower temperature and higher density. These length scales are much smaller than the characteristic sizes of structures featuring in the coronal models under discussion, and the timescales are much shorter than those of coronal phenomena except transient flare phenomena. Furthermore, the plasma length scales are strongly anisotropic because of the effects of the magnetic field. Gradients parallel to the field, in which direction the mean free path is long, tend to be much weaker than in the perpendicular direction. Under typical coronal conditions, the MHD approximation is therefore appropriate. For a more detailed discussion on MHD in general we refer to Braginskii (1965) and for applying MHD to the coronal plasmas to Petrie (2000) and Marsch (2006).

Figures 2 and 3 show examples of global coronal MHD models compared with observations of coronal magnetic structures. The MHD model includes a self-consistent description of the structure of both the magnetic field and the plasma. Synthetic images can therefore be derived from the model plasma state parameters, and these can be directly compared with observations. An example is shown in Fig. 2, where a synthetic image for the coronal polarization brightness, based on a steady-state MHD model, is compared with an eclipse photograph. Also shown are selected field lines from the model. It is clear that bright features in the white-light corona correspond to regions with closed magnetic field in the model.

Synthetic EUV images can also be derived from MHD models, for comparison with EUV observations of the corona from, e.g., SoHO/EIT, STEREO/SECCHI/EUVI or SDO/AIA. Figure 3 shows a comparison of observations from SDO/AIA in four different wavelength (171 $\mathrm{A}, 193 \AA, 211 \AA, 335 \AA$ ) with synthetic images created from a thermodynamic solution of an MHD-model.

This exercise illustrates the degree to which the MHD model plasma state variables are under-constrained by the observations. On the other hand, insight can be gained regarding the heating distribution in the corona: it can be argued that the heating model producing the coronal hole distribution in best agreement with observations is closest to the real heating distribution of the corona.

Solving the full MHD equations on a sizable three-dimensional grid requires computational resources beyond a present-day personal workstation. Also, MHD models are difficult to constrain observationally, since there are many free parameters representing quantities that are difficult to measure. Fortunately, MHD is not necessary for all coronal applications, and there are further useful approximations where models can be produced more easily and cheaply, and constrained only by surface magnetic field measurements.

The first such approximation to consider is the steady-state approximation, where temporal variations are ignored. Because the characteristic speed of the corona is high, the corona tends to evolve in a quasi-static manner, punctuated by episodes of abrupt dynamical change. It is the equilibrium states that we are interested in here. The Alfvén speed in the corona is estimated to be around $1000 \mathrm{~km} \mathrm{~s}^{-1}$ or higher (Aschwanden 2005). For an active region of size 200 or $300 \mathrm{Mm}$ the Alfvén transit time is a few to five minutes. Therefore, if a region's magnetic structure remains essentially unchanged over timescales significantly longer than five minutes, e.g., on timescales of order $1 \mathrm{hr}$, then the magnetohydrostatic approximation becomes relevant. 
Fig. 2 Comparison between the predicted solar magnetic field lines from an MHD model (top panel) and corona polarization brightness (middle panel) for the 2008 August 1 total solar eclipse from an MHD model and the sharpened white-light eclipse image (bottom panel) from Bor Udzuur, Mongolia. The images are oriented with solar north $12^{\circ} .1$ counterclockwise from the vertical direction. It is evident that the bright features in the white-light corona correspond to regions with closed magnetic field lines. From Rušin et al. (2010), Fig. 5. Reproduced with permission of authors
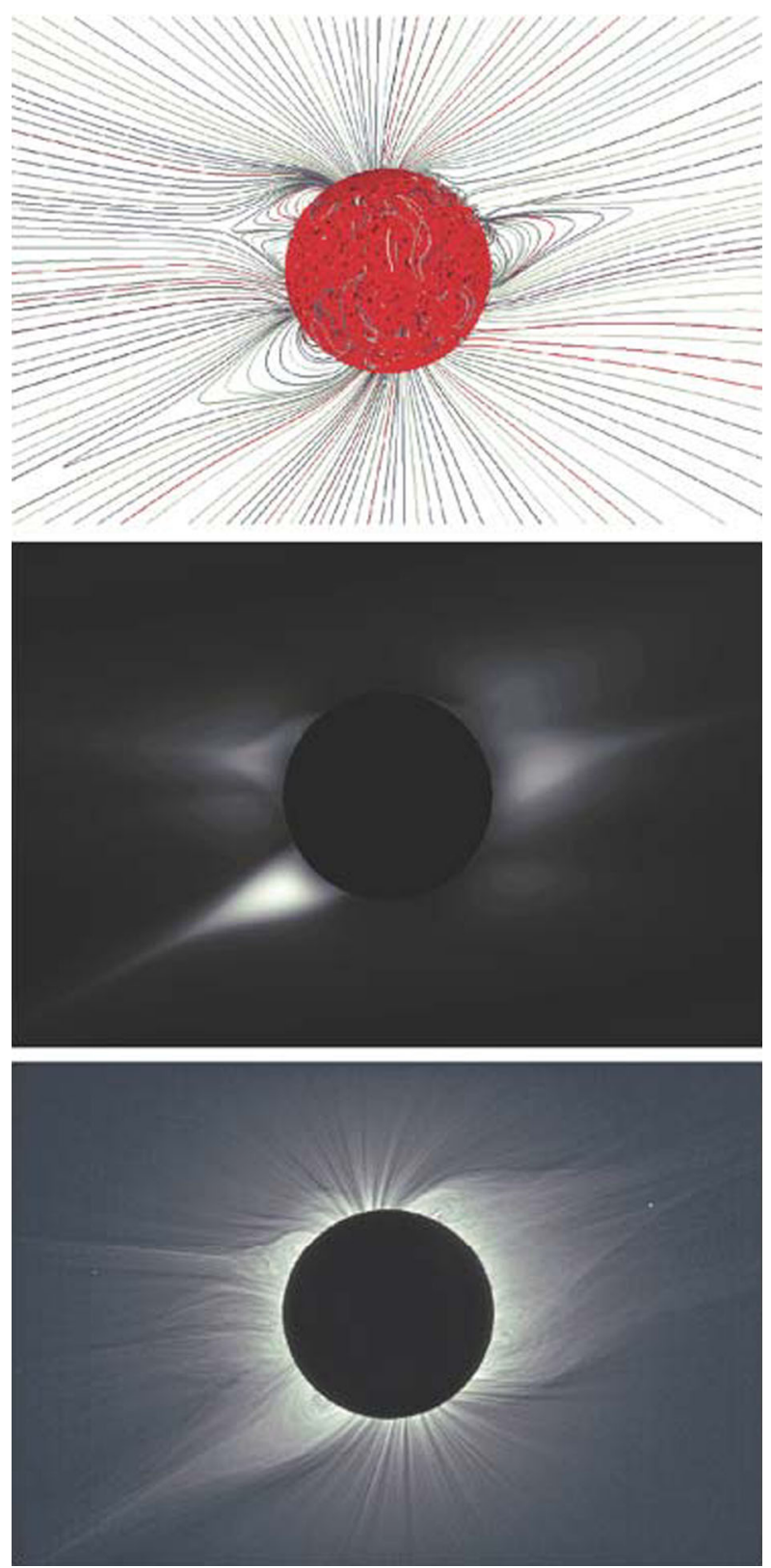

To quantify the relative influence of the time-dependent versus the time-independent terms, and the terms associated with the magnetic field versus those associated with the plasma, it is helpful to define some dimensionless constants as follows. The Alfvén Mach number $M=v / v_{A}$ gives the flow speed in terms of the Alfvén speed $v_{A}=B_{0} /\left(4 \pi \rho_{0}\right)^{1 / 2}$ for typical field strength $B_{0}$ and density $\rho_{0}$. The plasma beta $\beta=8 \pi p_{0} / B_{0}^{2}$, the ratio of the plasma pressure $p_{0}$ and magnetic pressure $B_{0}^{2} / 8 \pi$, measures the dominance of the mag- 


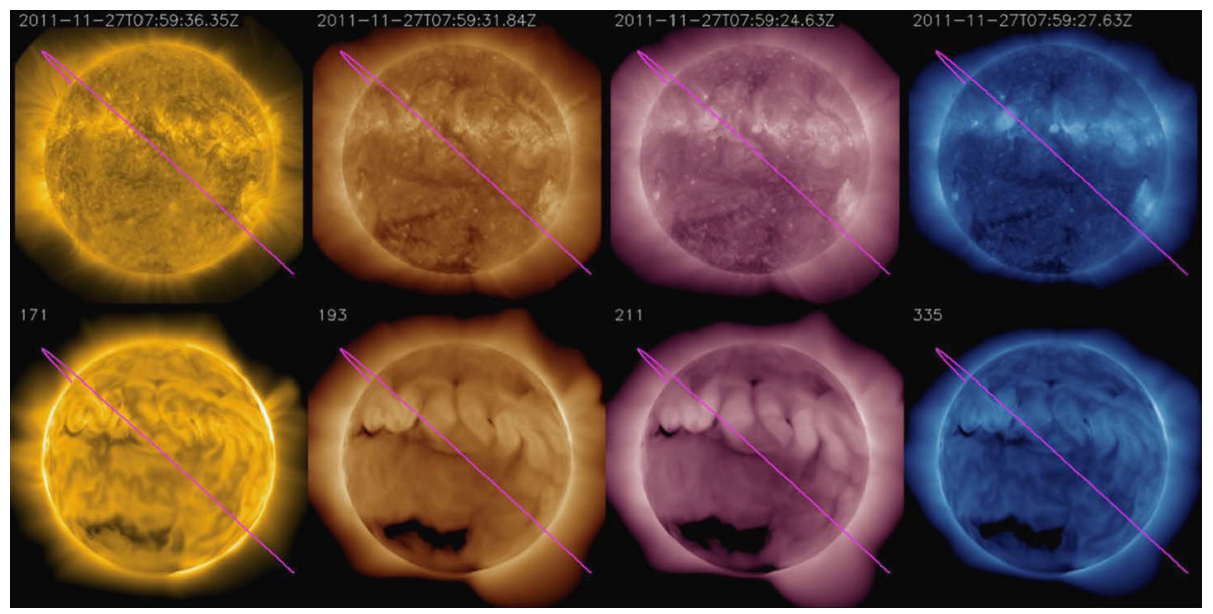

Fig. 3 A comparison of observations from SDO/AIA in different wavelength taken on November 27th 2011 (top panels) with synthetic images created from an MHD-model. From Downs et al. (2013), Supplementary material S3. The images where created as part of a study to investigate a Sun-Grazing Comet. A discussion of this topic is well outside the scope of this review, however. Reproduced with permission of authors

netic field. We define a similar ratio between the gravitational energy density and magnetic pressure, $\beta_{g}=8 \pi \rho_{0} \psi_{0} / B_{0}^{2}$, for $g=\nabla \psi$, where $\psi$ is the gravitational potential.

For length scale $L$ and Alfvén time $t_{A}=L / v_{A}$, the MHD equations normalize as,

$$
\begin{aligned}
\tilde{\rho}\left(\frac{t_{A}}{t_{0}} \frac{t_{0}}{t_{A}} \frac{\partial \tilde{\mathbf{v}}}{\partial \tilde{t}}+\frac{v_{0}^{2}}{v_{A}^{2}} \tilde{\mathbf{v}} \cdot \tilde{\nabla} \tilde{\mathbf{v}}\right) & =\tilde{\mathbf{j}} \times \tilde{\mathbf{B}}-\frac{\beta}{2} \tilde{\nabla} \tilde{p}-\frac{\beta_{g}}{2} \tilde{\rho} \tilde{\nabla} \tilde{\psi}, \\
\frac{L}{t_{0} v_{0}} \frac{\partial \tilde{\mathbf{B}}}{\partial \tilde{t}} & =\tilde{\nabla} \times(\tilde{\mathbf{v}} \times \tilde{\mathbf{B}}), \\
\frac{L}{t_{0} v_{0}} \frac{\partial \tilde{\rho}}{\partial \tilde{t}}+\tilde{\nabla} \cdot(\tilde{\rho} \tilde{\mathbf{v}}) & =0, \\
\tilde{\nabla} \cdot \tilde{\mathbf{B}} & =0 .
\end{aligned}
$$

In Eqs. (1)-(4), tildes indicate normalized variables.

\section{Magnetohydrostatic Models}

If flows are sub-Alfvénic, i.e.,

$$
\frac{t_{A}}{t_{0}}=\frac{v_{0}}{v_{A}}=\varepsilon \ll 0,
$$

then Eq. (1) becomes,

$$
\mathbf{0}=\tilde{\mathbf{j}} \times \tilde{\mathbf{B}}-\frac{\beta}{2} \tilde{\nabla} \tilde{p}-\frac{\beta_{g}}{2} \tilde{\rho} \tilde{\nabla} \tilde{\psi},
$$

and Eqs. (2), (3) become trivial. Equation (6) is the magnetohydrostatic equation, describing the balance between the Lorentz force and the plasma pressure gradient and gravitational forces. 


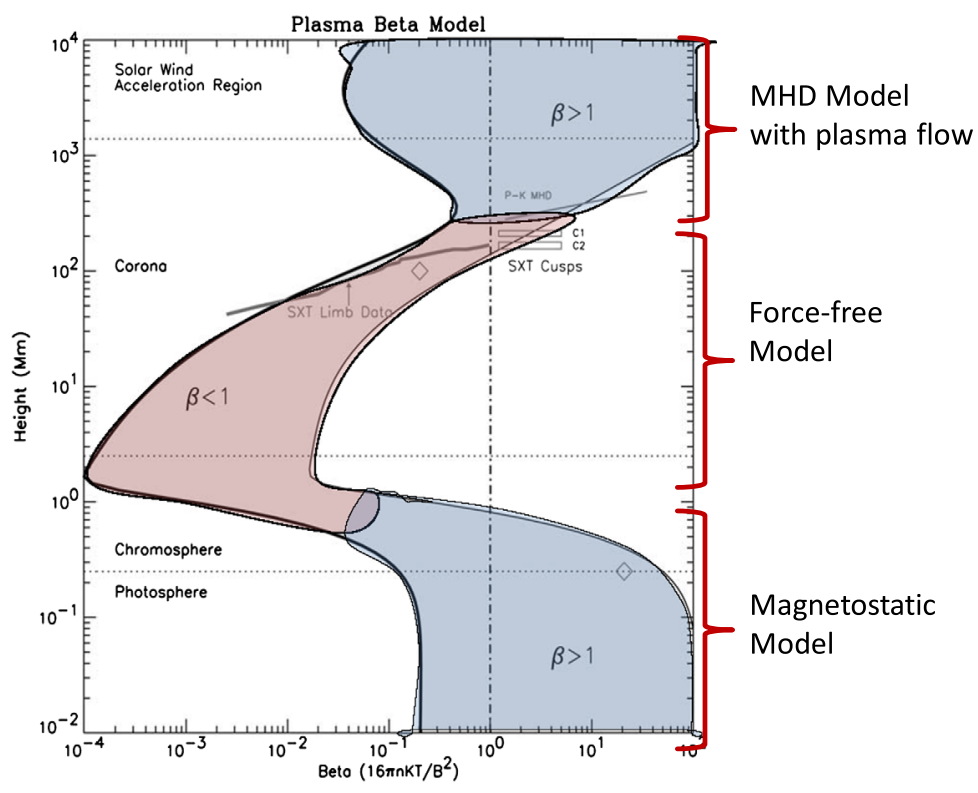

Fig. 4 Plasma $\beta$ in the solar atmosphere. Modified version from Gary (2001), Fig. 3. Reprinted with kind permission of Springer Science and Business

While the corona contains a low plasma $\beta$, this is not the case in the lowest layers of the solar atmosphere (photosphere and lower chromosphere) and also not in the area beyond the source surface at about $2.5 \mathrm{Rs}$, see also Sect. 4 and Fig. 4 . Consequently non-magnetic forces like pressure gradients, gravity and the ram pressure of the solar wind have to be taken into account for this regions. Here we briefly discuss magneto-hydro-static approach (no plasma flows), which is a reasonable assumption for flow velocities below the Alfvén and sound speed.

In the generic, non-linear case, it is in principle possible to solve the MHS-equation (6) by minimizing a functional

$$
L(\mathbf{B}, p, \rho)=\int\left[\frac{|(\nabla \times \mathbf{B}) \times \mathbf{B}-\nabla p-\rho \nabla \Psi|^{2}}{B^{2}}+|\nabla \cdot \mathbf{B}|^{2}\right] d V
$$

and derive iterative equations for the magnetic field $\mathbf{B}$, plasma pressure $p$, and plasma density $\rho$ (see Wiegelmann et al. 2007, for details). The MHS-optimization principle generalizes (and has it heritage) from optimization principles for nonlinear force-free fields, see Sect. 4. For complete and consistent boundary conditions this optimization approach works well and reconstructs analytic test solutions with high accuracy, but significant slower convergence time compared with force-free approaches. In particular mixed plasma $\beta$ regions lead to long computing times, because $\mathbf{B}, p$, and $\rho$ have to be iterated simultaneously and low plasma $\beta$ regions with small (but non-vanishing) plasma forces require strongly reduced iteration time steps.

Somewhat more popular (but less realistic) are MHS-approaches with further restrictions, e.g. on the electric current density in the form:

$$
\nabla \times \mathbf{B}=\alpha_{0} \mathbf{B}+f(r)(\nabla(\mathbf{r} \cdot \mathbf{B})) \times \mathbf{r} .
$$


In this approach the electric current contains a linear force-free part $\alpha_{0} \mathbf{B}$ and a current on spherical shells strictly perpendicular to gravity. $\alpha_{0}$ is the global constant linear force-free parameter and $f(r)$ a free function. An advantage of representing the current in the form of Eq. (8) is that it linearizes the mathematical problem and separable solutions have been found in different geometries. Naturally (global) solar applications have some preference for spherical geometry (see Bogdan and Low 1986, for solutions without the linear forcefree part) and (Neukirch 1995, with both parts of the current). A principal limitation of this approach is that splitting the electric current in this form is motivated by mathematical simplicity (one aims to find separable solutions) and not by physical reasoning. The forcefree parameter $\alpha_{0}$ has to be globally constant, which is not a very realistic assumption for modelling the entire corona (but might be reasonable for localized regions) and also the free function $f(r)$ has to be globally fixed, which causes additional limitations. Linear MHS models are moderately popular (see, e.g., Bagenal and Gibson 1991; Gibson and Bagenal 1995; Gibson et al. 1996; Zhao and Hoeksema 1994; Zhao et al. 2000; Ruan et al. 2008, for solar applications), but the amount of studies based on this model is much lower compared to the simpler PFSS-models described in Sect. 5. Separable solution in cartesian geometry have been computed by Fourier transform (Low 1991) and Greens function methods (Petrie and Neukirch 2000).

\section{Force-Free Models}

It is well known that the magnetic field dominates the plasma in the corona, unlike in the photosphere where the dense plasma dominates the dynamics (except arguably in sunspots), or in the heliosphere where the solar wind is dominant. Gary (2001) constructed a simple one-dimensional model for the magnetic stratification of the solar atmosphere and constrained the magnetic and plasma pressures of this model using numerous observations at various heights, see Fig. 4. He argued that there is a range of heights in the solar atmosphere, between the chromosphere and about $100 \mathrm{Mm}$, where the plasma $\beta \ll 1$. Within this height range, the magnetic field is so dominant in the force balance that the effect on the field structure of the interaction between the Lorentz force and the plasma forces is negligible, i.e., the force-free approximation can apply.

Thus in the magnetically dominated corona, the plasma pressure and gravity forces can be neglected, so that Eq. (6) becomes $\tilde{\mathbf{j}} \times \tilde{\mathbf{B}}=\mathbf{0}$, resulting in the force-free equation,

$$
\nabla \times \mathbf{B}=\alpha(\mathbf{x}) \mathbf{B},
$$

where the spatially-dependent scalar function $\alpha(\mathbf{x})$ scales as $1 / L$. The function $\alpha(\mathbf{x})$ may be interpreted as the magnetic twist per unit length. Since, from Eq. (4) and the divergence of Eq. (9), $\mathbf{B} \cdot \nabla \alpha=0, \alpha$ is constant along magnetic field lines. The force-free model is referred to as the linear force-free field (LFFF) model if $\alpha$ is globally constant, and as the nonlinear force-free field model (NLFFF) if $\alpha$ varies from field line to field line.

Since $\alpha(\mathbf{x})$ scales as $1 / L$, this parameter is unimportant for large structures, and significant $\alpha$ values are confined to closed fields within active regions, such as the filament labeled in Fig. 5. In force-free models, non-zero $\alpha$ represents Maxwell stresses trapped within the field in the form of twist or shear. Such a field is not in its lowest energy state: in principle, the Maxwell stress can be released in an eruption such as a flare or CME, fueled by the free magnetic energy contained in the stressed field. The force-free model is the minimal requirement for reconstructing the coronal field of active regions with significant free magnetic energy. 

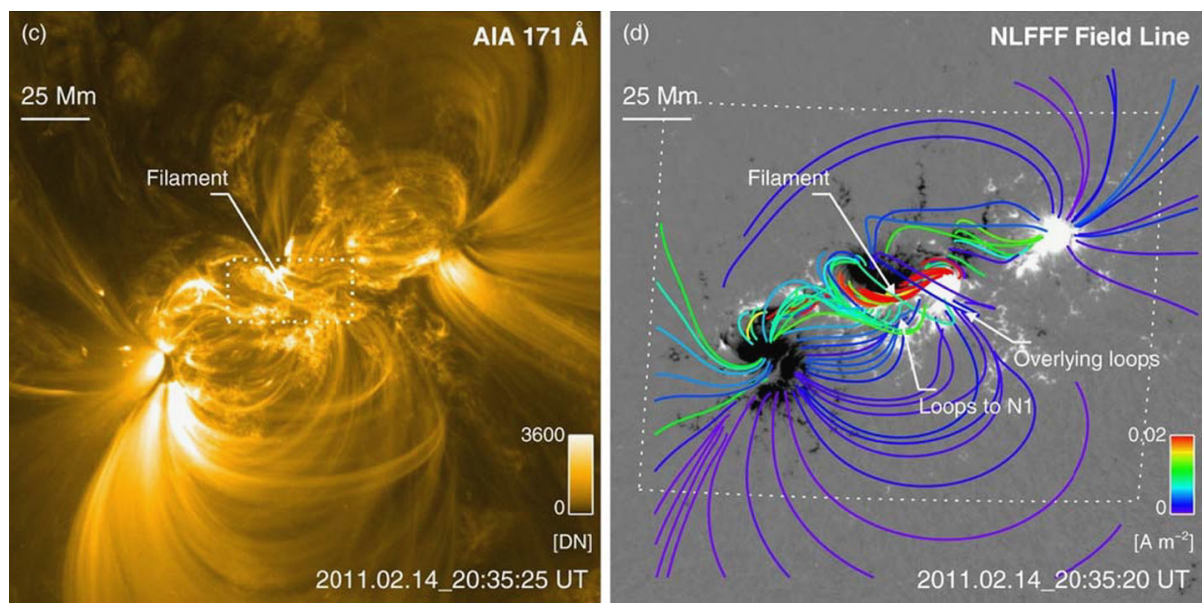

Fig. 5 Observations and modeling results for AR 11158 on 2011 February 14 20:35 UT, about 5 hr before the X2.2 flare. Left: Image from AIA 171-band showing the corona magnetic structures. Right: Selected field lines from the NLFFF extrapolation plotted over a cutout from the vertical field map. The lines are color-coded by the vertical current density at their footpoints (see the color bar); red field lines correspond to strong current density. [From Sun et al. (2012), Fig. 1. (C) AAS. Reproduced with permission]
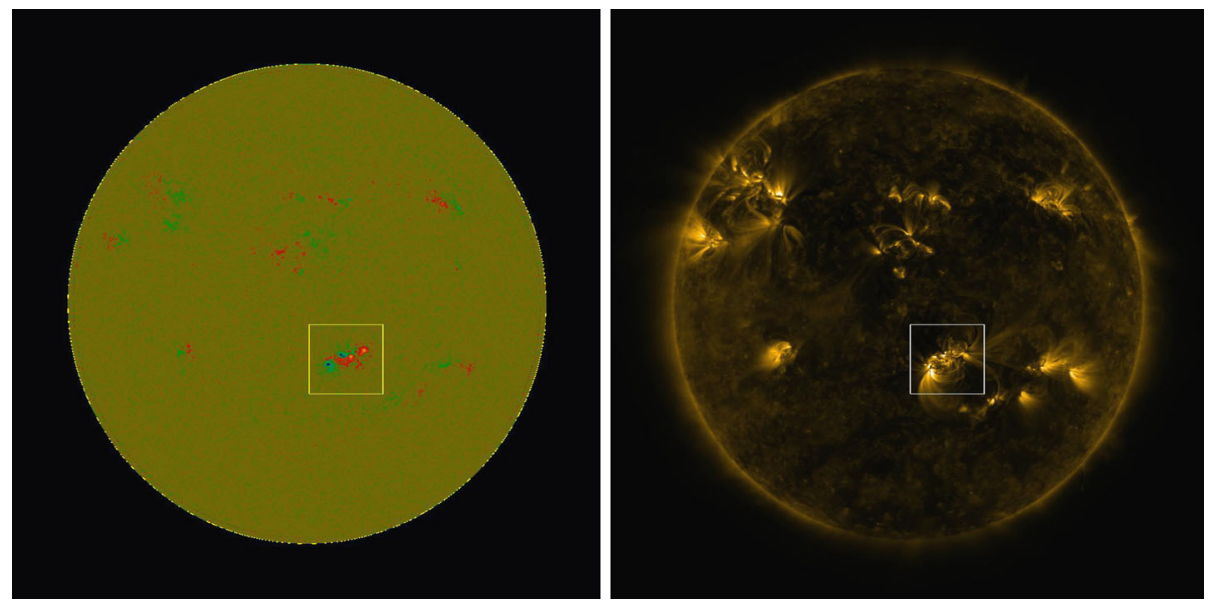

Fig. 6 The left panel shows a magnetogram from SDO/HMI and the right panel a coronal image from SDO/AIA. The marked area corresponds to an active region which was reconstructed with a cartesian force-free code. It takes typical about 5-10 h to compute a NLFFF-model for an active region on a desktop PC. [Source: Wiegelmann et al. (2012), Fig. 1. Reprinted with kind permission of Springer Science and Business]

The force-free approximation allows us to extrapolate a solution from measurements in the lower atmosphere independently of the state of the plasma. The force-free model has no model for the plasma structure, and so it is usually validated by comparing chosen model field lines with observed magnetic structures in, e.g., EUV images such as the examples shown in Figs. 5 and 6. Since full disk vector magnetograms are routinely available from 
Fig. 7 Global nonlinear force-free coronal magnetic field model based on a synoptic vector magnetogram from SOLIS (June 2012). Green and red lines correspond to open and closed field lines. The background image shows the coronal plasma observed with SDO/AIA.

[Source: Fig. 2 in Tadesse et al. (2014a). Reprinted with kind permission of Springer Science and Business Media]

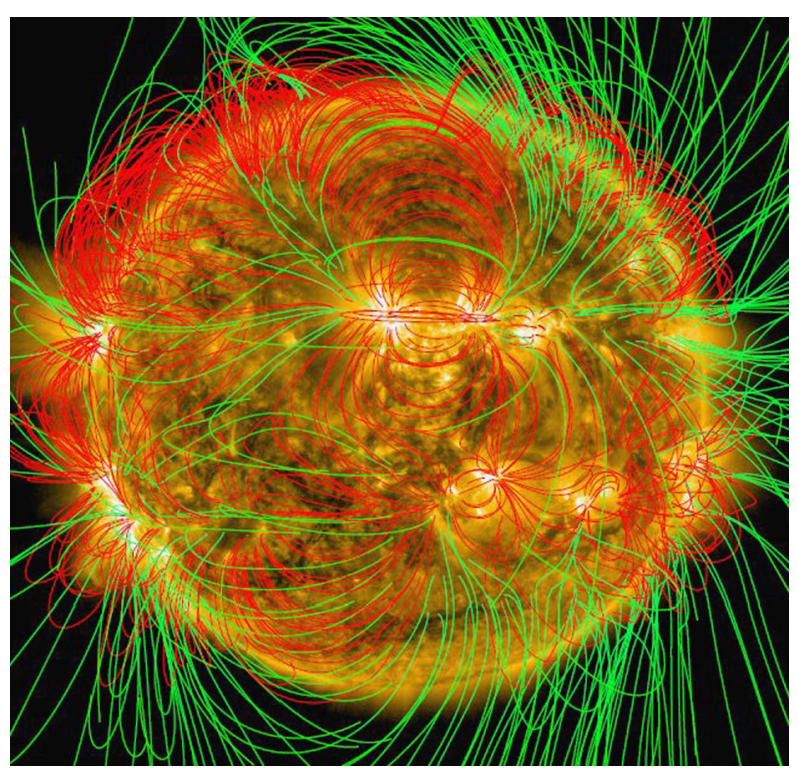

SDO/HMI, NLFFF models are available also on global scales, see Fig. 7. While the numerical more expensive NLFFF models resemble the observed structure (e.g. as visible in SDO/AIA) somewhat better than simpler potential field models (see Tadesse et al. 2014c, for a comparison of both models). The study by Tadesse et al. (2014c) revealed that active regions are magnetically connected, but hardly share a significant amount of electric currents. A study by Tadesse et al. (2014b) based on synoptic vector magnetograms from SOLIS, revealed that the total magnetic energy for the global NLFFF model was about $10 \%$ than in a PFSS model and the free magnetic energy was located mainly within active regions. Consequently NLFFF models for the global coronal field tend to be close to potential fields, except within active regions. Figure 8 shows a global NLFFF model by Amari et al. (2014), where the global field is untwisted and unsheared, even though there is a highly twisted flux rope present in the model. Because the force free function $\alpha$ is inversely dependent on length scale, large scale (or global) structures are hardly influenced by moderate changes of $\alpha$. This illustrates why potential field models have been successful in modeling the global coronal magnetic field.

\subsection{Methods for Computing Nonlinear Force-Free Fields}

Here we briefly describe different methods how non-linear force-free coronal magnetic fields can be computed. For earlier reviews on force-free fields see Amari et al. (1997); Wiegelmann (2008); Wiegelmann and Sakurai (2012) and an overview of magnetic fields in the upper solar atmosphere has been given recently in Wiegelmann et al. (2014).

The force-free equations are defined by a vanishing Lorentz-force leading to

$$
\begin{aligned}
(\nabla \times \mathbf{B}) \times \mathbf{B} & =\mathbf{0}, \\
\nabla \cdot \mathbf{B} & =0,
\end{aligned}
$$


Fig. 8 Top: Set of field lines of the reconstructed global solar force-free magnetic field. The calculation is performed with resolution $171 \times 251 \times 363$ and use active-region and full-disk magnetograms from SDO/HMI, and synoptic map from SOLIS. Bottom: The structure at the active region shown in the plot of the global solution in the left picture. A twisted flux rope in equilibrium is clearly seen to be present. [From Amari et al. (2014), Figs. 2 and 3.

Reproduced with permission of authors]
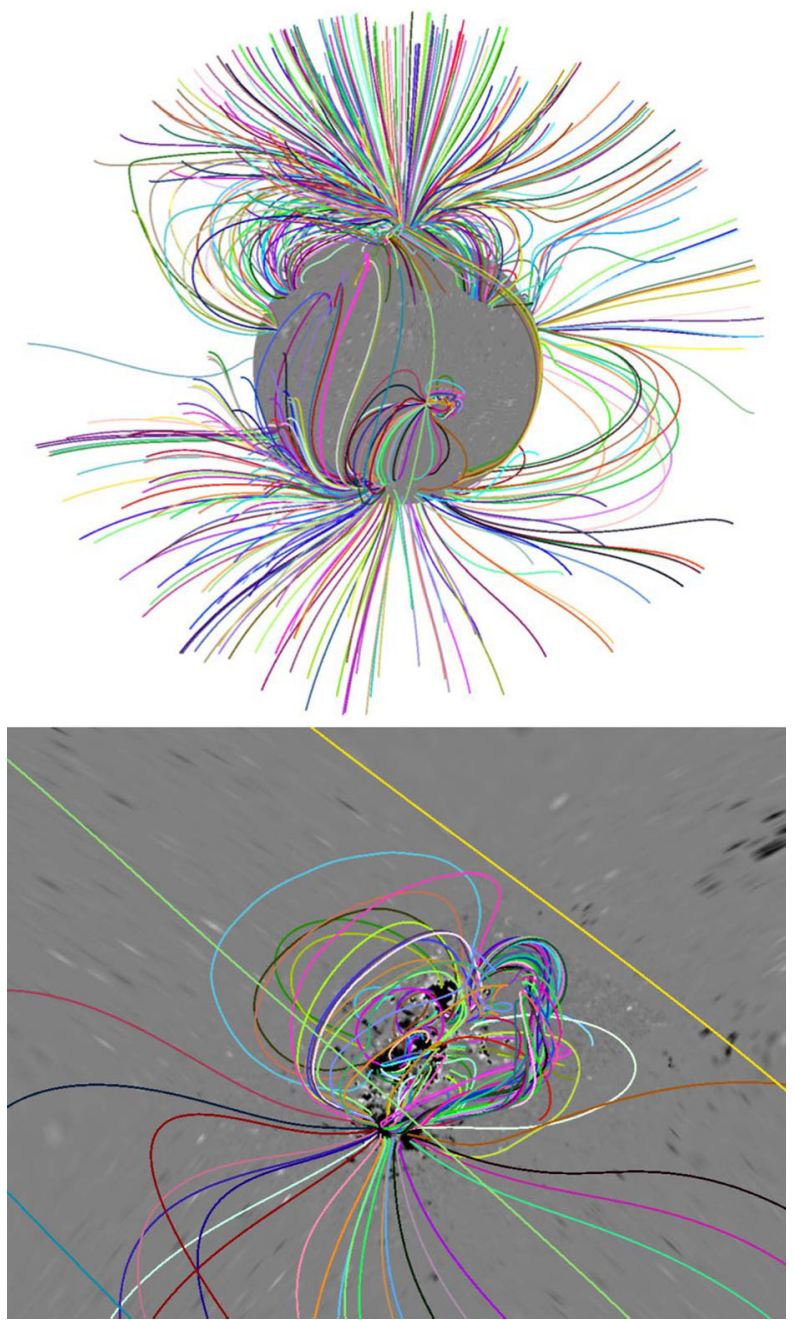

where $\mathbf{B}$ is the magnetic field. This set of equations is equivalent to

$$
\begin{aligned}
\nabla \times \mathbf{B} & =\alpha \mathbf{B}, \\
\mathbf{B} \cdot \nabla \alpha & =0,
\end{aligned}
$$

where the force-free function $\alpha$ is constant along magnetic field lines. These sets of equations, either (10) and (11) or (12) and (13) are solved numerically with the measured magnetic field vector in the photosphere as boundary condition. As a first step for a force-free model a current-free potential field is computed, which requires only the vertical magnetic field component as boundary condition. This potential field model is then used as an initial equilibrium before numerical schemes include electric currents. As boundary condition one uses either the photospheric magnetic field vector more or less directly (usually after some preprocessing) and solves Eqs. (10) and (11) by MHD-relaxation or optimization. Alternatively, one uses only the vertical photospheric field and the vertical current density (the ratio 
of these quantities corresponds to the distribution of $\alpha$ on the photosphere) as boundary conditions and solves (12) and (13) with Grad-Rubin codes. Other approaches, like direct upward integration and the boundary element methods use both the photospheric field vector and the distribution of $\alpha$. Current joint approaches of the nonlinear force-free consortium (a group of scientists comparing, testing and evaluating NLFFF models since 2004) concentrate on Grad-Rubin, MHD-relaxation and optimization, however and so do we in this review. See Wiegelmann (2008) and references therein for the upward integration and the boundary element methods.

\subsection{Grad-Rubin Codes}

The Grad-Rubin approach, originally proposed by Grad and Rubin (1958) for fusion plasmas, uses the vertical magnetic field and the $\alpha$ distribution for one polarity (positive or negative) as boundary condition. As initial condition a potential field is used and the electric current (or $\alpha$ ) is injected into the computational domain by solving Eq. (13). This naturally changes the magnetic field structure as re-computed from Eq. (13). The iterative process continues until a stationary state is reached. Mathematicians like the Grad-Rubin very much, because it is based on a well posed mathematical problem (see, e.g., Amari et al. 2006) and at least for moderate values and gradients of $\alpha$ it has been shown that a solution exists and is unique (Bineau 1972). For $\alpha$ derived from measurements, which can have high values and strong gradients due to localized current concentrations convergence of the Grad-Rubin method is, however, not ensured. An additional pitfall is that one has two distinct well posed problems: (1) vertical magnetic field and vertical current computed from the positive polarity and (2) vertical magnetic field and vertical current computed from the negative polarity. And the solutions of these two problems usually differ significantly for measured data (see, e.g., Schrijver et al. 2008). In a subsequent study Wheatland and Régnier (2009) showed that it is possible to extend the Grad-Rubin code by an outer iteration circle, which computes the photospheric $\alpha$ distribution from both polarities and includes the measurement errors of the horizontal magnetic field to weight between $\alpha$-values computed from both polarities.

\subsection{MHD-Relaxation and Optimization}

These code have many similarities. Starting from a potential field, they solve the force-free equations by iterating

$$
\frac{\partial \mathbf{B}}{\partial t}=\mu \mathbf{F}
$$

where the pseudo force $\mathbf{F}$ is derived from a reformulation of the MHD-equations (relaxation codes, see, e.g., Valori et al. 2005) or from minimizing a functional similar as in the MHS case (7), but here the plasma terms vanish (optimization codes, see, e.g., Wheatland et al. 2000).

As initial condition the computational box contains a potential field, which is then disturbed by an injection of the measured photospheric magnetic field vector. Originally both approaches fixed the magnetic field vector on the lower boundary of the computational box, but newer codes allow now a more gradual introduction of these boundary conditions and allow to change the horizontal field on the boundary in accordance with measurement errors. The iteration of Eq. (14) continues until a stationary state is reached and the pseudo force $\mathbf{F}$ vanishes. A principal problem with the original approach (magnetic field vector fixed on the lower boundary) is that for measured data, which include noise and inconsistencies, a solution of (10) and (11) cannot be guaranteed. Preprocessing of the measurements reduces but 


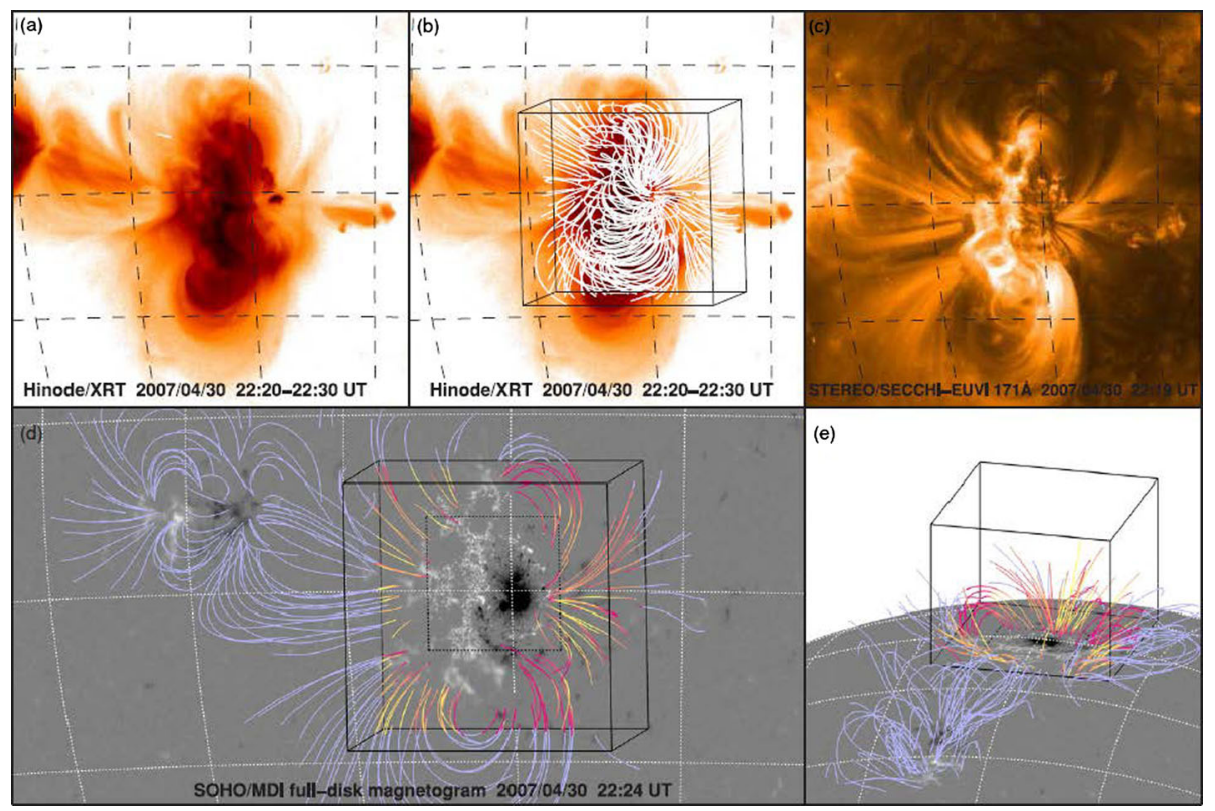

Fig. 9 Comparison of NLFFF-models and stereo-loops. Panel a shows a Hinode soft X-ray image of AR10953, in panel b overlaid with NLFFF-fieldlines. Panel c depicts one of the STEREO-SECCHI images, which has been used for stereoscopy. Finally in panels $\mathbf{d}$ and $\mathbf{e}$ the stereoscopic loops (in blue) are compared with NLFFF loops. The dotted area depicts the region where photospheric vector field measurements from Hinode have been available, the solid black lines the NLFFF computational domain (outside the HinodeFOV only LOS-measurements from SOHO/MDI have been available). [Source: DeRosa et al. (2009), Fig. 1. (C) AAS. Reproduced with permission]

does not entirely solve this problem. Optimization codes have been developed in cartesian and spherical geometry, see Figs. 7 and 8 for global spherical NLFFF-models based on an optimization code and a Grad-Rubin method, respectively.

\subsection{Comparison and Evaluation of NLFFF-Codes}

In a series of workshops/publications a group of scientists have compared and evaluated NLFFF-codes since about a decade-henceforth referred to as the NLFFF consortium. The comparison with synthetic data (for which the exact solutions where known, see Schrijver et al. 2006; Metcalf et al. 2008) revealed that the codes are very reliable and accurate if fed with complete and consistent boundary conditions. Complications occur, when the boundary conditions are neither complete, nor force-free consistent, which is unfortunately the case for a number of photospheric measurements (see also Sect. 7). For such inconsistent boundary conditions (a relative small FOV measured by Hinode) the model fields differed in geometry, magnetic energy content, and degree of force-freeness (see Schrijver et al. 2008). In a subsequent study (DeRosa et al. 2009) coronal magnetic field lines obtained from NLFFF-models have been compared with stereoscopically reconstructed coronal loops, see Fig. 9. The comparison was not satisfactory. NLFFF models did not perform better than potential fields in this study. Possible reasons are the small FOV of the vector data from Hinode, which only covered about $10 \%$ of the area spanned by the stereoscopic reconstructed loops. Also the separation angle of the two spacecraft was very small $\left(7^{\circ}\right)$ which leads to a large stereo- 
scopic reconstruction error. DeRosa et al. (2009) concluded that a successful application of NLFFF models requires:

1. Large model volumes at high resolution that accommodate most of the connectivity within a region and to its surroundings;

2. Accommodation of measurement uncertainties (in particular in the transverse field component) in boundary condition;

3. Preprocessing of the lower-boundary vector field for a realistic approximation of the high-chromospheric, near force-free field;

4. Force-free models should be compared (or even improved) with coronal observations

Boundary conditions for large model volumes are routinely available since the launch of SDO in 2010 and recent updates of NLFFF-codes are suitable to incorporate measurement errors (see Wheatland and Leka 2011, for Grad-Rubin codes and Wiegelmann and Inhester 2010, for optimization). We will discuss preprocessing in Sect. 7, which is dedicated to boundary conditions (for all methods, not limited to NLFFF). Let us note that some datasets of SDO/HMI are remarkably force-free consistent in the photosphere and do not require preprocessing (Wiegelmann et al. 2012). A comparison of extrapolation results with coronal images (like SDO/AIA) is now routinely performed, see Fig. 7. We briefly address recent methods to improve NLFFF models with coronal observations in Sect. 7.

\section{Potential-Field Models for the Global Coronal Field}

When we neglect electric currents from the coronal model, we can represent the field using a scalar potential, $\mathbf{B}=\nabla \phi$, so that Eq. (4) leads to a Laplace equation

$$
\nabla^{2} \phi=0
$$

with the line-of-sight or normal derivative of $\phi$ determined on the lower boundary by the observational line-of-sight or radial field component (Altschuler and Newkirk 1969; Schatten et al. 1969). Force-free electric currents can be significant in active regions, as we have seen in Figs. 5 and 8. The other major location where significant electric currents are likely to flow is the volume above the low- $\beta$ region in the corona, corresponding to the volume above heights of order $100 \mathrm{Mm}$ in the model of Gary (2001). At these heights the thermal and dynamical pressures of the expanding solar wind can exceed the coronal magnetic pressure, under which conditions the fields cannot return to the Sun. Instead they are dragged out into the heliosphere. In the PFSS model (Altschuler and Newkirk 1969; Schatten et al. 1969), this effect of the solar wind on the coronal field is modeled by setting the scalar potential to a constant value on an outer boundary surface, called the source surface, forcing the model field to be radially directed there. The radius of the source surface is usually set to between 2 and 3 solar radii (Hoeksema 1984). Within the source surface the field is dominant and current-free. Outside the source surface the field is dominated by the solar wind and follows the spiral trajectory of this wind.

Equation (15) can be solved analytically as an expansion of spherical harmonics (Schatten et al. 1969; Altschuler and Newkirk 1969) or numerically using a finite difference approach (e.g. Riley et al. 2006). In a recent study Tóth et al. (2011) compared the spherical harmonic decomposition method with a finite difference approach and the authors found significant differences. A spherical harmonic decomposition is prone to artifacts like rings around strongly localized structures. Another problem is that polar field measurements are 

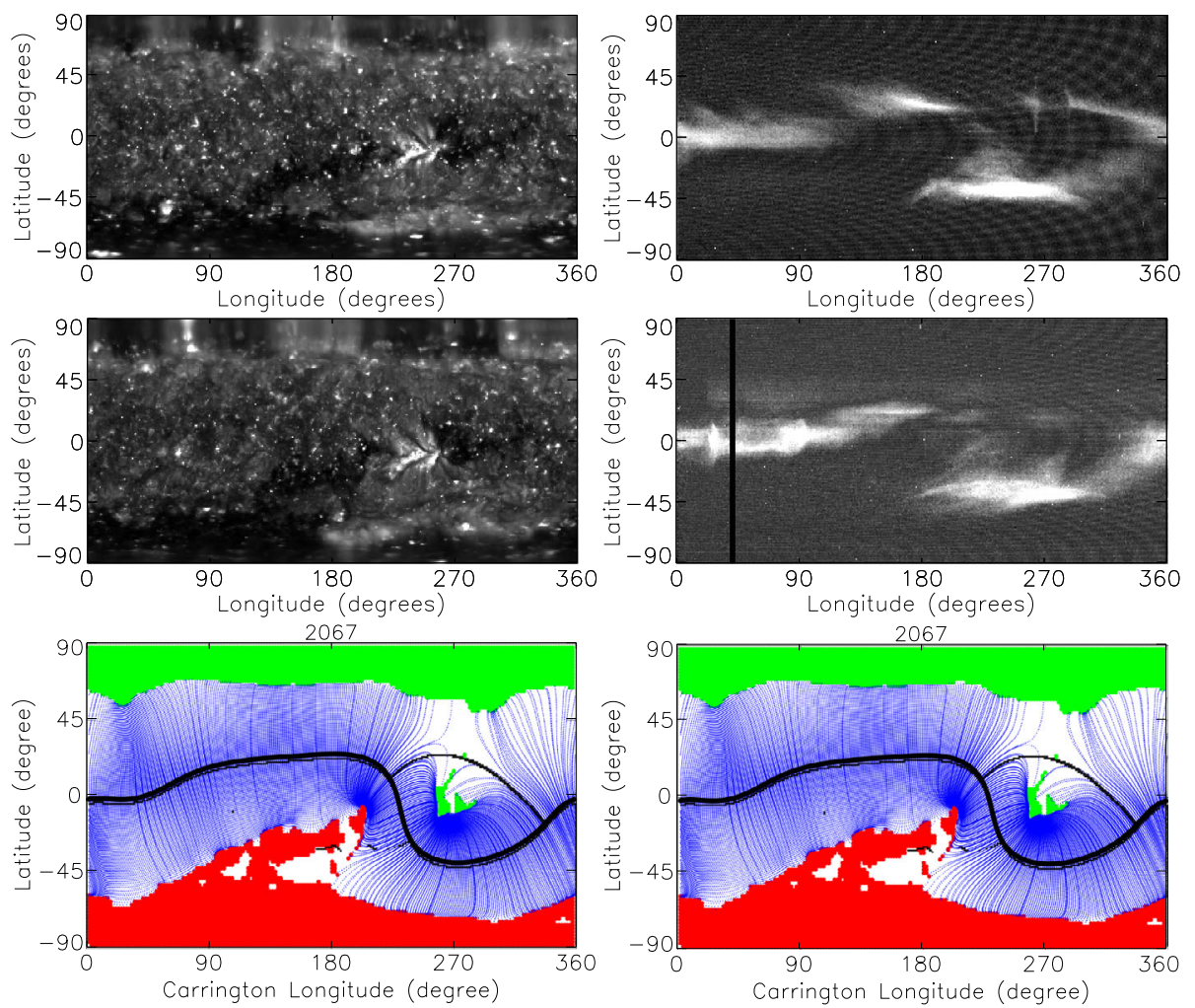

Fig. 10 STEREO/SECCHI/EUVI 171 A synoptic maps for Carrington rotation 206, from the ahead (top left) plot and behind (middle left plot) spacecraft. STEREO/SECCHI/COR1 synoptic maps for Carrington rotation 2067 from the ahead (top right plot) and behind (middle right plot) spacecraft. These maps show the streamer brightness at 2.6 solar radii off the east limb. PFSS models extrapolated from the GONG synoptic magnetograms Carrington rotations 2067 (bottom pictures, which are identical). Positive and negative model coronal holes are colored red and green. The streamer-belt neutral lines are represented by thick black lines and pseudo-streamer locations by thin black lines. Streamer-belt fields are plotted in blue. [From Petrie et al. (2011). Figs. 2, 3 and 4. Reprinted with kind permission of Springer Science and Business Media]

difficult because of, among other things, the very large projection angle associated with the small angle between the solar rotation axis and the ecliptic plane, the fact that each pole is visible for $<6$ months each year, the intrinsic weakness of the polar fields, and limb noise. These difficulties are discussed by Petrie et al. (2014). For both numeric and analytic PFSS methods, the radial magnetic field at the inner boundary is derived from observed photospheric magnetic field measurements (typically by using precomputed synoptic maps). At the outer radial boundary (typically $1.5-3.5 R_{S}$ ), the field is forced to become radial. This outer boundary is, in effect, a tunable parameter in the model, allowing the modeler to better match observed features in the corona (e.g., the location of coronal hole boundaries) or the solar wind (the amount of open flux inferred from interplanetary spacecraft).

Though the PFSS model is simple, its phenomenology is rich. Selected classes of field lines correspond to well-known large-scale coronal features. Open field lines represent coronal holes, and the set of footpoints of open fields can be compared to coronal hole observations in EUV or He I $10830 \AA$ A. The set of tallest closed field lines can be compared to the 
main streamer belt and the set of pseudostreamers seen in coronagraph images. Comparisons of both coronal hole and streamer locations are shown in Fig. 10.

Here we have only mentioned the most popular methods for reconstructing the coronal magnetic field structure from photospheric or chromospheric field measurements. Other methods include the extension of the PFSS model to include a heliospheric current sheet by Schatten (1971), and the linear magnetohydrostatic approach (8) is a generalization of the PFSS model and shares with it the possibility of a spherical harmonic decomposition. For global computations based on magnetofrictional simulations see Yeates and Mackay (2012).

\section{Comparison Between PFSS and MHD Models}

As we have noted, the large-scale, quasi-static magnetic field within the corona is computed using PFSS, NLFFF, MHS or MHD models. All techniques rely on measurements of the photospheric magnetic field (line-of-sight field or vector field). In the following we want to compare the simplest (PFSS) and most sophisticated approach (MHD) in some detail.

PFSS models have the advantage of being simple to develop and apply, and do not require significant computational resources (Riley et al. 2006). Additionally, they are, in principle, capable of resolving spatial scales beyond those currently seen with MHD solutions. However, they are limited because their basic assumptions are rarely, if ever met in reality. In particular: (1) magnetic fields in the low corona often depart significantly from a potential configuration; (2) time-dependent effects (e.g., reconnection) are likely important processes that reconfigure the magnetic field; and (3) the reference surface at which the field becomes radial is generally not spherically-shaped. In the following subsections, we summarize the PFSS and MHD techniques, and explore each of these limitations in more detail.

MHD models can be thought of as a refinement (albeit substantial) to the PFSS approach. They solve the full set of resistive MHD equations, typically in spherical coordinates, and can incorporate a wide range of physical processes. Most recently, self-consistent descriptions of waves/turbulence have been incorporated with the aim of prescribing both the heating of the corona and the acceleration of the solar wind in a self-consistent fashion (e.g. Lionello et al. 2014). For the purposes of comparing with PFSS results, however, we limit ourselves to the simpler polytropic version of the model, in which the energy equation is simplified by an adiabatic equation, where the polytropic index, $\gamma$, is set to 1.05 , to mimic the near isothermal properties of the corona. Although this results in plasma values and variations that do not match well with observations, the magnetic structure of the corona appears to be well captured.

The MHD equations have been described in Sect. 2 and the methods used to compute the MHD solutions can be found in Mikić and Linker (1994), Lionello et al. (1998), Riley et al. (2006). Here, we note only that: (1) the coronal model computes solutions between $1 R_{S}$ and $20-30 R_{S}$; (2) we use the same radial photospheric magnetic field to drive the MHD model; and (3) although the solutions are time-dependent, we advance the solution forward in time until a steady-state equilibrium is reached.

Figure 11 compares the MHD and PFSS solutions for two Carrington rotations representative of solar minimum (CR1910) and solar maximum (CR1969) conditions. In each panel, the same starting points were used to trace the field lines; thus, differences in the location and shape of the field lines represent differences in the model solutions. We infer that the two approaches appear match reasonably well, at least qualitatively, at both solar minimum and maximum. However, there are several noteworthy differences. For example, the PFSS model does not reproduce the cusp-like features of the streamer belt, which merge into the 


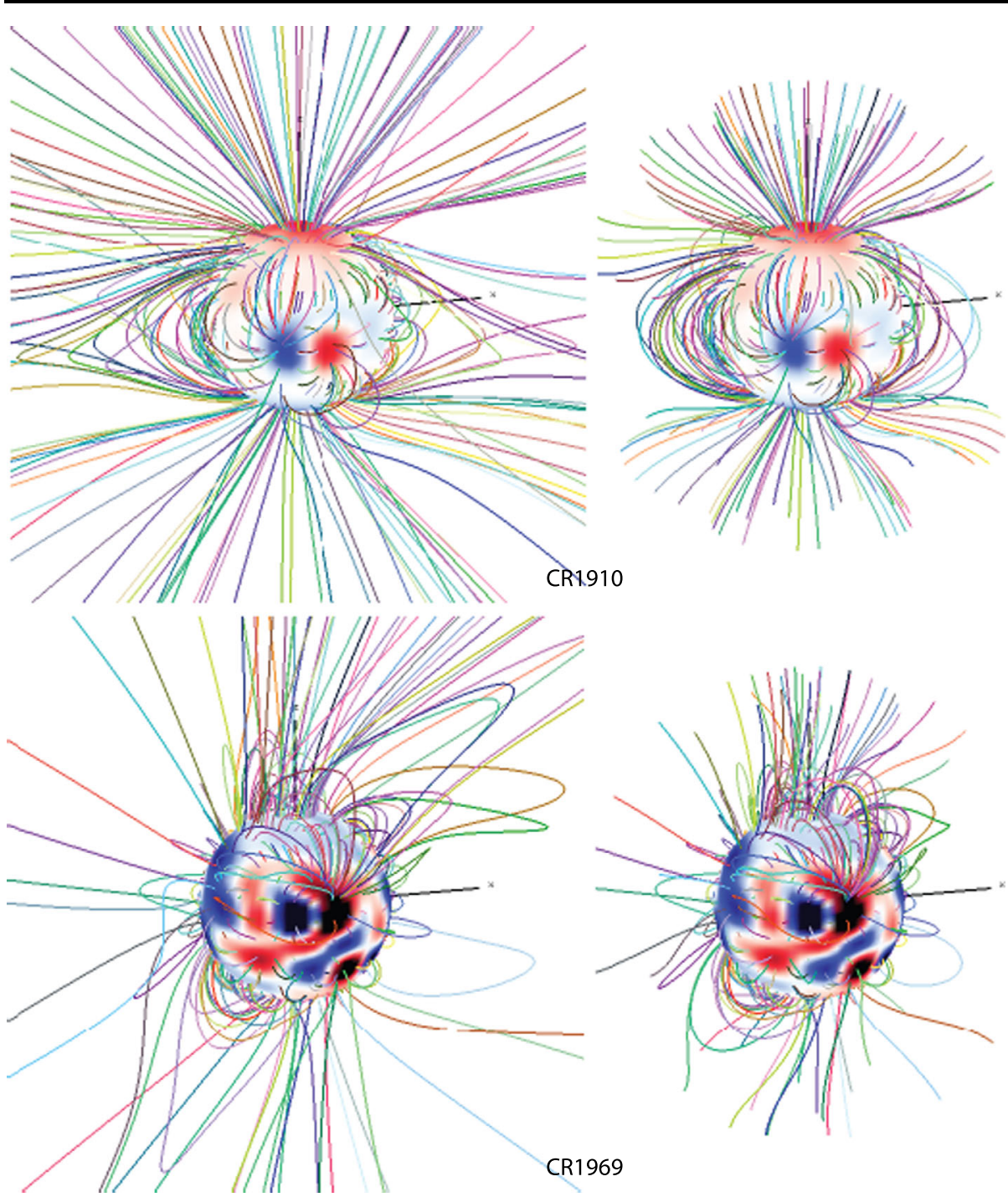

Fig. 11 Comparison between MHD solution (left) and PFSS model (right) for two solar rotations illustrative of solar minimum (CR1910) and solar maximum (CR1969) conditions. The solar surface is colored according to the radial component of the magnetic field at the photosphere. Field lines have been colored arbitrarily for clarity. [Adapted from Riley et al. (2006), Fig. 1. (C) AAS. Reproduced with permission]

heliospheric current sheet in the MHD solution. Additionally, the PFSS model appears to underestimate the amount of open solar flux, as inferred from the total number of field lines that open up into the heliosphere. Finally, in general, field lines in the PFSS solutions are shorter than their MHD counterparts.

\subsection{Is the Source Surface Really Spherical?}

One of the basic assumptions of the PFSS model is that, at the outer radial boundary, the field lines are radial, i.e., this surface is a spherical equipotential. Figure 12 tests this assumption. 
(a)

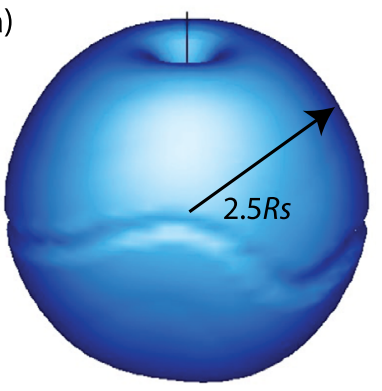

(b)
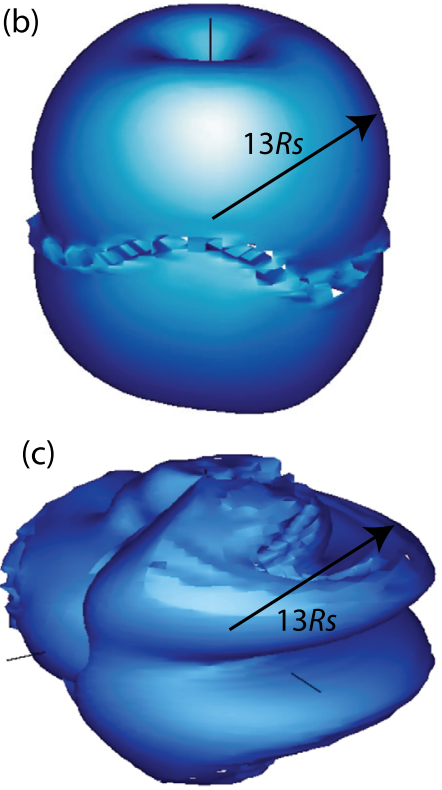

(d)
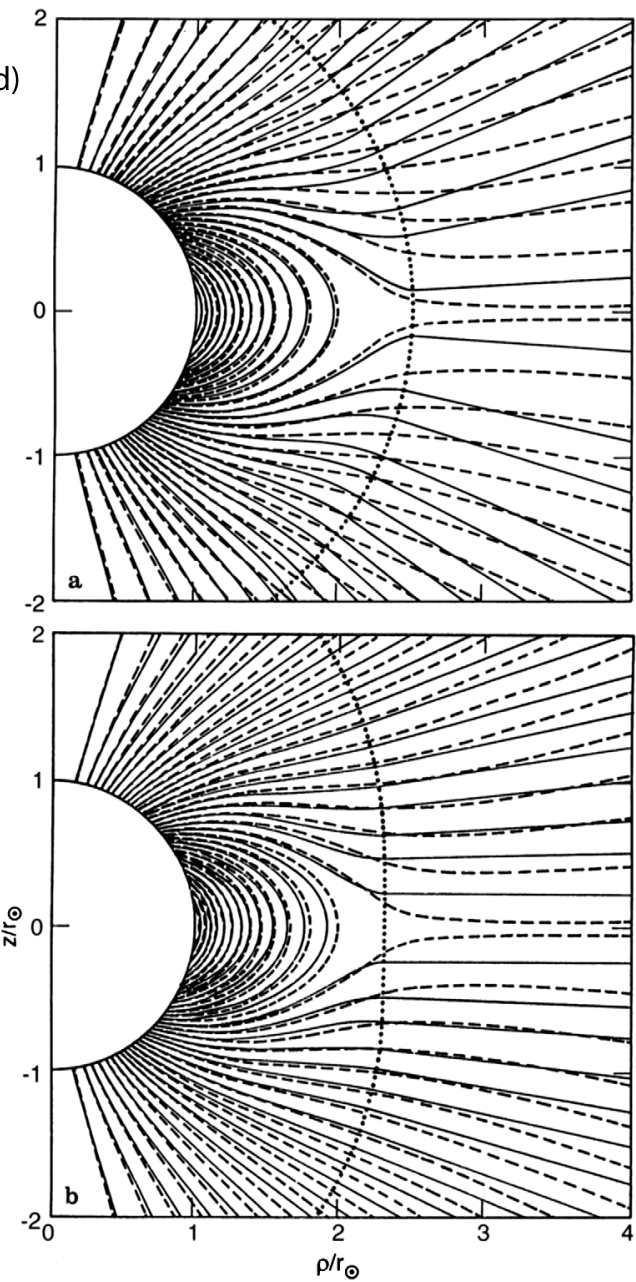

Fig. 12 Isosurfaces of $\left|B_{r}\right| /|B|=0.97$ for: (a) PFSS solution for CR1910; (b) MHD solution for CR1910; and (c) MHD solution for CR1969. The Sun's rotation axis is indicated by the vertical black line. (d) PFSS solutions using a spherical source surface (top) and "dipole-isogauss" source surface (bottom). The dashed lines in both panels of (d) correspond to MHD model solutions. (a)-(c) were adapted from Riley et al. (2006), Figs. 4 and 5. (d) [from Schulz et al. (1978), Figs. 1 and 2. Panels a-c: (C) AAS. Reproduced with permission; Panel d: Reprinted with kind permission of Springer Science and Business Media]

In panel (a), we have plotted the isosurface of $\left|B_{r}\right| /|B|=0.97$ for the PFSS solution of CR1910. Not surprisingly, the field lines have become radial by $2.5 R_{S}$. The sinusoidal variation about the equator marks the boundary between outwardly and inwardly directed fields. Although there are no current sheets in the PFSS model, this corresponds to where the neutral line would lie. The polar dimples illustrate the fact that while the model forces the field lines to become radial by $2.5 R_{S}$, there is no requirement that it cannot occur closer to the Sun, as it does when there are large unipolar regions. Panels (b) and (c) show the same isosurface for the MHD solutions at solar minimum and maximum. We note that the field lines do not become substantially radial until beyond $10 R_{S}$. Moreover, at solar minimum, the shape of the source surface is prolate spheroid, again with dimples at the poles. At solar 

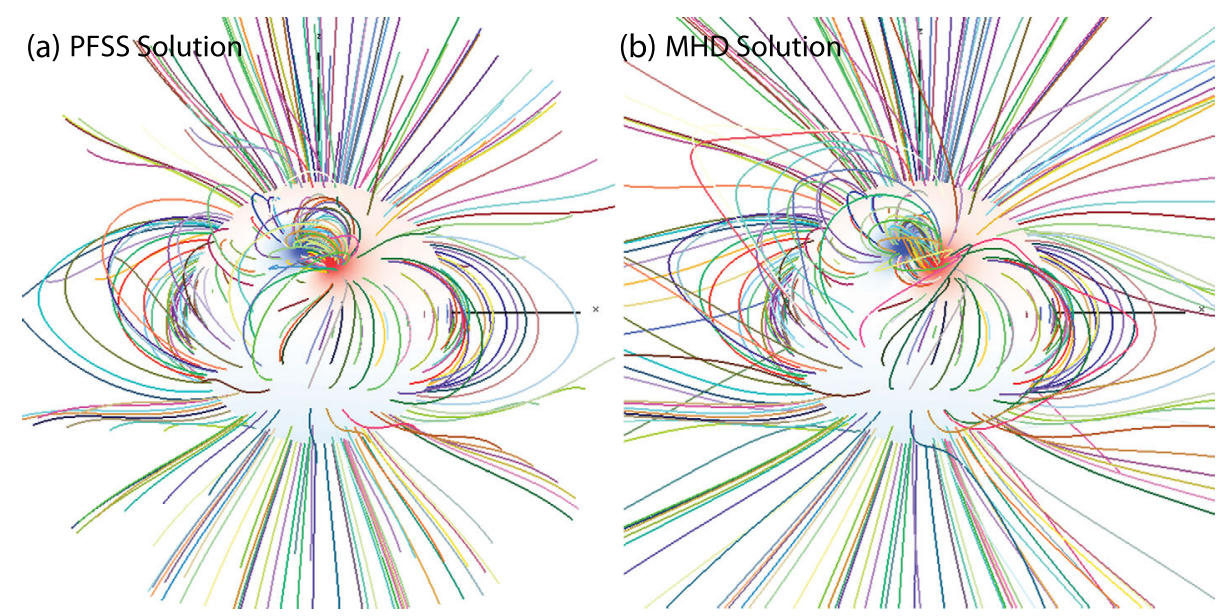

Fig. 13 (a) PFSS and (b) MHD solutions for an idealized large-scale dipole configuration with an embedded active region. See text for more details. [Adapted from Riley et al. (2006), Figs. 8 and 9. ๑ AAS. Reproduced with permission]

maximum, the isosurface displays a more complex structure that changes from one rotation to the next. On average, however, it could be argued that it tends to be more spherical. The main point, however, is that the MHD model implies that the Sun's real 'source surface' is located further away than the canonical 2.5 $R_{S}$ used in PFSS models.

Figure 12(d) summarizes two analytic PFSS solutions relying on (top) a spherical source surface and (bottom) a "dipole-isogauss" (or prolate spheroid) isosurface. The first of these matches the shape in panel (a) while the latter more closely resembles (b). Thus, we conclude that even during solar minimum conditions an important, yet simple refinement to PFSS models is to employ a "dipole-isogauss" surface, as suggested by Schulz et al. (1978) for axisymmetric cases.

\subsection{Is the Field Really Potential?}

To explore the possible effects of non-potentiality on the solutions, we extracted a snapshot from an idealized time-dependent MHD solution, in which an active region was being emerged. We computed a PFSS solution using the radial magnetic field at the base of the MHD solution and compared the results with the MHD model (Fig. 13). The comparison is somewhat artificial in the sense that had we re-run the MHD model with this radial boundary condition, allowing it to reach a steady-state equilibrium, the comparison between the PFSS and MHD solutions would have been more similar to that in Fig. 11. However, our point is to better understand the differences that non-potentiality can introduce into the solutions. We remark that the overall, large-scale features are qualitatively similar. The MHD fields tend to be more inflated, and, hence, more opened. The largest closed fields in the MHD solution also display a cusp-like geometry, whereas the PFSS solution does not. The most striking differences, however, are related to the active region, where the PFSS solution has not reproduced any of the sheared magnetic field lines that are aligned with the neutral line, which separates the two polarities of the bipole. 
(a) PFSS Solutions

WS Model

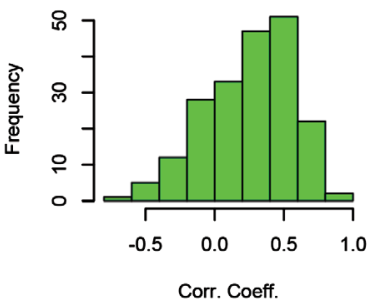

(b) MHD Solutions

WS Model

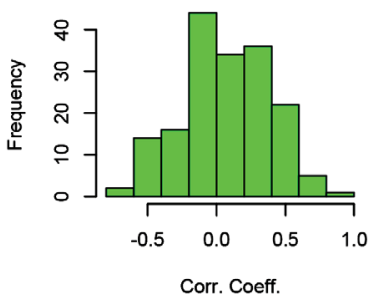

DCHB Model

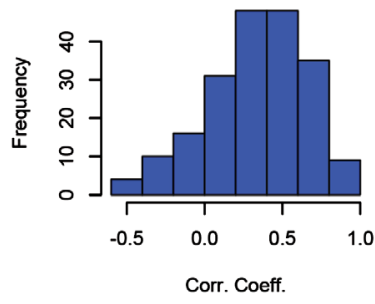

DCHB Model

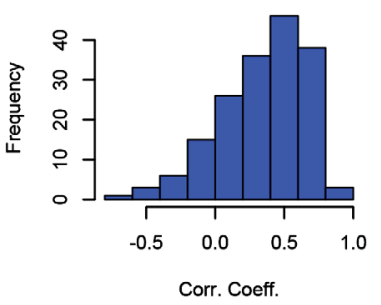

WSA Model

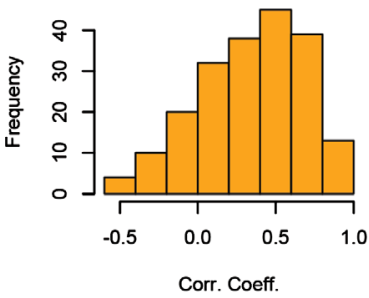

WSA Model

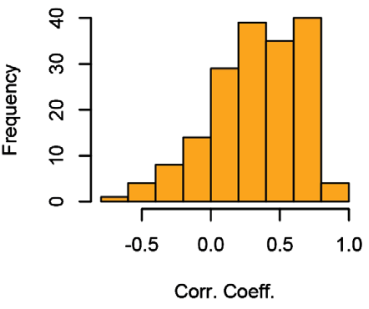

Fig. 14 Histograms of Pearson correlation coefficients of modeled and observed solar wind speed at $1 \mathrm{AU}$ in the ecliptic plane for an interval running from 1996 through 2011. (a) Results when the model is driven by a PFSS solution, using (left) the WS model, (middle) the DCHB model, and (right) the WSA model. (b) The same empirical models are compared when driven by MHD solutions. See text for more details. [Adapted from Riley et al. (2015). Reproduced with permission of authors]

\subsection{The PFSS and MHD Approaches in Action: Predicting Solar Wind Speed}

Riley et al. (2015) developed a set of empirically-based models to better understand the relationship between solar wind speed models. In particular, they compared the predicted speeds of solar wind plasma at $1 \mathrm{AU}$ in the ecliptic plane over a 15-year period. They found that, in general, a model relying exclusively on the expansion factor of the coronal magnetic fields to estimate the speed of the plasma (Wang and Sheeley 1990), did not perform as well as a 'boundary layer' model, where the speed of the solar wind was everywhere fast, except in a band at the edge between open and closed field lines (Riley et al. 2001). Of relevance here, they also found that the expansion factor model performed significantly better when it was driven by a PFSS model as oppose to an MHD solution. Since, based on the fact that the MHD model has generally been found to produce a better match with a wide range of remote solar observations, this suggests that the expansion factor and PFSS techniques are somehow conspiring together to produce better results.

Figure 14 compares Pearson correlation coefficients estimated by comparing modeled and observed solar wind speed, rotation by rotation, over a period of 15 years. In each row, the coefficients are compared for the WS (expansion factor), DCHB (boundary layer), and WSA (hybrid approach) techniques. When the PFSS model is used in all three cases, the correlations are comparable. When the MHD solutions are instead used, the DCHB and WSA correlations improve modestly, but the PFSS correlations drop dramatically. This can be understood in terms of the assumptions of the PFSS model. Specifically, by requiring that the field become radial by $2.5 R_{S}$, the modeled flux tubes do not have the correct properties, 
particularly near the boundary between open and closed field lines, where the expansion factors are larger over a wider distance from the open/closed boundary.

In conclusion, the PFSS model continues to offer a simple method for computing largescale coronal magnetic fields under certain conditions, and subject to some limitations. However, as computers become more capable, and global MHD algorithms become generally more available to the scientific community, their advantages may be outweighed by their limitations.

\subsection{Possible Improvements of MHD Models}

Global MHD models of the solar corona are continually being refined and improved. These developments can be categorized in terms of numerical improvements or scientific improvements. The former, include the ability to run larger runs, using more processors, allowing us to explore finer spatial scales at increased temporal resolution. Additionally, as new computational hardware becomes available, such as GPUs, additional leaps into new regimes may be possible. While these developments can and should lead to a better understanding of the physical processes at work, it is through the incorporation of better physics that we expect to learn the most. Currently, coronal models are run in either the so-called polytropic or thermodynamic approximations: The latter allows a more realistic treatment of energy transport processes, but still relies on ad hoc prescriptions for the heating of the corona and acceleration of the solar wind (Riley et al. 2012a, 2012b; Riley and Luhmann 2012). Most recently, models incorporating self-consistent descriptions of wave-turbulence (Cranmer et al. 2007, e.g.) have been developed (Lionello et al. 2014). These promise to shed new insight to the underlying mechanisms that heat the corona and accelerate the solar wind. Additionally, models now can address multiple species, with different gyrotropic temperatures (van der Holst et al. 2014, e.g.).

\section{A Note on Boundary Conditions}

One inescapable fact of extrapolating coronal field models from photospheric measurements is that the physical conditions of the high- $\beta$ photosphere do not match those of the low- $\beta$ corona. This means that direct application of observed photospheric data as coronal model boundary data is not a physically consistent approach. The photospheric field is frozen into a high- $\beta$ plasma that dominates its dynamics, tending to keep it far from a force-free state, whereas the coronal plasma is low- $\beta$ and the coronal field nearly force-free. Though many photospheric fields, e.g., sunspot penumbrae or flaring neutral-line fields, are nearly horizontal, most photospheric fields have been found to be approximately vertical to within about $10^{\circ}$ (Svalgaard et al. 1978; Wang and Sheeley 1992; Petrie and Patrikeeva 2009; Gosain and Pevtsov 2013). For this reason, computing the radial field component $B_{r}$ from the line-of-sight component $B_{\mathrm{LOS}}$ as $B_{r}=B_{\mathrm{LOS}} / \cos \left(\rho_{\mathrm{h}}\right)$, where $\rho_{\mathrm{h}}$ is the heliospheric angle, gives a reasonable estimate for the radial flux distribution over the photospheric surface. This gives a continuous $B_{r}$ component, but discontinuous tangential components $\mathbf{B}_{\mathrm{h}}$, across the photospheric surface. The discontinuity is interpreted as a transition from the nearly radial, non-potential photospheric field to the nearly potential, non-radial coronal field: the transition region is mathematically idealized as a current layer (Wang and Sheeley 1992). This radial-field approximation has become an essential part of the standard method for applying line-of-sight photospheric data as boundary data for PFSS and MHD models, including those in Figs. 2, 3 and 10. It not only makes more coherent physical sense than applying 
Fig. 15 The original concept of NLFFF-modeling from photospheric measurements (blue boxes) and future plans to incorporate chromospheric measurements from Solar-C (red)

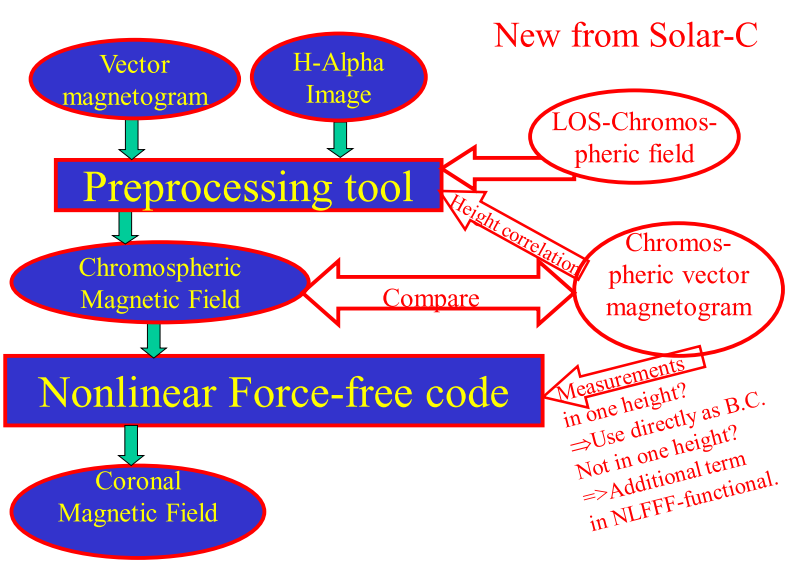

line-of-sight measurements directly as boundary data for the line-of-sight field component of the model, but also results in more successful models (Wang and Sheeley 1992).

For the NLFFF model, the problem of applying photospheric boundary data as lowercoronal boundary conditions is more complex. The NLFFF model involves solving the vector equation (9) (and $\boldsymbol{\nabla} \cdot \mathbf{B}=0$ ), and so the solution is over-constrained if we impose the three observed photospheric vector field components as boundary conditions. In this sense the problem is mathematically ill-posed. There also remains the problem that the measurements derive from the high- $\beta$ photosphere, and are therefore associated with significant Lorentz forces in general, whereas the coronal model is force-free by assumption. In practice, the boundary data are modified to be approximately force-free (Wiegelmann et al. 2006, 2008) or are reduced to boundary data for $B_{z}$ and $(\nabla \times \mathbf{B})_{z} / B_{z}$ to form a mathematically well-posed problem with boundary conditions consistent with a force-fee field. Recently, methods have been developed that ignore the horizontal photospheric field information and instead use intensity patterns in chromospheric or coronal images to guide the modeling (Aschwanden 2013; Malanushenko et al. 2014). In a recent approach (Chifu et al. 2015) demonstrated how photospheric vector magnetograms and stereoscopic coronal observations can be combined within the NLFFF approach. This new approach does not ignore the horizontal photospheric field, but stabilizes the NLFFF computation for cases where the field measurements contain large measurement uncertainties.

\subsection{Use Chromospheric Measurements, e.g., from Solar-C}

As seen in Fig. 4 the plasma- $\beta$ in the photosphere is about unity or more and consequently the photospheric magnetic field is not necessarily force-free. For force-free coronal magnetic field extrapolations it would be preferable to measure the field at the base of the corona or in the upper chromosphere, where $\beta$ is small. Currently we estimate the field in the upper solar chromosphere by a preprocessing of photospheric measurements, which allows also the incorporation of direct chromospheric observations like H- $\alpha$ images (Wiegelmann et al. 2008) and the use of line-of-sight chromospheric fields as measured with Solis (Jing et al. 2010). It would be preferable, however, to measure the chromospheric magnetic field vector directly, as planned for the Solar-C mission. A concept how these measurements can be incorporated into NLFFF-modeling is shown in Fig. 15. A problem is that the exact height above the photosphere of the chromospheric measurements is a priori unknown and one needs to incorporate a correlation analysis with extrapolations from the photosphere to specify this height. 


\subsection{Incorporate Coronal Measurements}

Direct coronal measurements have carried out for magnetic sensitive coronal lines. Several presentations have been given on the ISSI-2015 meeting and corresponding publications are planned in the same special issues as this paper, e.g. by Phil Judge, Stephen White, Roberto Casini on Coronal measurements and by Javier Trujillo Bueno, Egidio Landi degl-Innocenti on UV Polarized Radiation Observables for Probing the Upper Chromosphere and Corona. Due to the optical thin coronal plasma these measurements have a line-of-sight integrated character and require vector tomography methods (see Kramar et al. 2006, 2013) to derive the 3D coronal magnetic field structure. A prosperous approach is to combine extrapolation methods from photospheric measurements with a vector tomographic inversion of coronal measurements, because algorithms for both can be derived from optimization principles.

Acknowledgements We acknowledge warm hospitality and fruitful discussions during a workshop on Solar Magnetic Fields: From Measurements Towards Understanding at ISSI in Bern in January 2015.

Open Access This article is distributed under the terms of the Creative Commons Attribution 4.0 International License (http://creativecommons.org/licenses/by/4.0/), which permits unrestricted use, distribution, and reproduction in any medium, provided you give appropriate credit to the original author(s) and the source, provide a link to the Creative Commons license, and indicate if changes were made.

\section{References}

M.D. Altschuler, G. Newkirk, Magnetic fields and the structure of the solar corona. I: methods of calculating coronal fields. Sol. Phys. 9, 131-149 (1969). doi:10.1007/BF00145734

T. Amari, J.J. Aly, J.F. Luciani, T.Z. Boulmezaoud, Z. Mikic, Reconstructing the solar coronal magnetic field as a force-free magnetic field. Sol. Phys. 174, 129-149 (1997). doi:10.1023/A:1004966830232

T. Amari, T.Z. Boulmezaoud, J.J. Aly, Well posed reconstruction of the solar coronal magnetic field. Astron. Astrophys. 446, 691-705 (2006). doi:10.1051/0004-6361:20054076

T. Amari, J.J. Aly, P. Chopin, A. Canou, Z. Mikic, Large scale reconstruction of the solar coronal magnetic field. J. Phys. Conf. Ser. 544(1), 012012 (2014). doi:10.1088/1742-6596/544/1/012012

M.J. Aschwanden, Physics of the Solar Corona. An Introduction with Problems and Solutions, 2nd edn. (2005)

M.J. Aschwanden, Nonlinear force-free magnetic field fitting to coronal loops with and without stereoscopy. Astrophys. J. 763, 115 (2013). doi:10.1088/0004-637X/763/2/115, arXiv:1212.2996

F. Bagenal, S. Gibson, Modeling the large-scale structure of the solar corona. J. Geophys. Res. 96, 17,663 (1991). doi:10.1029/90JA02625

M. Bineau, Existence of force-free magnetic-fields. Commun. Pure Appl. Math. 25, 77 (1972)

T.J. Bogdan, B.C. Low, The three-dimensional structure of magnetostatic atmospheres. II-Modeling the large-scale corona. Astrophys. J. 306, 271-283 (1986). doi:10.1086/164341

S.I. Braginskii, Transport processes in a plasma. Rev. Plasma Phys. 1, 205 (1965)

I. Chifu, B. Inhester, T. Wiegelmann, Coronal magnetic field modeling using stereoscopy constraints. Astron. Astrophys. 577, A123 (2015). doi:10.1051/0004-6361/201322548

S.R. Cranmer, A.A. van Ballegooijen, R.J. Edgar, Self-consistent coronal heating and solar wind acceleration from anisotropic magnetohydrodynamic turbulence. Astrophys. J. Suppl. Ser. 171, 520-551 (2007). doi:10.1086/518001, arXiv:astro-ph/0703333

M.L. DeRosa, C.J. Schrijver, G. Barnes, K.D. Leka, B.W. Lites, M.J. Aschwanden, T. Amari, A. Canou, J.M. McTiernan, S. Régnier, J.K. Thalmann, G. Valori, M.S. Wheatland, T. Wiegelmann, M.C.M. Cheung, P.A. Conlon, M. Fuhrmann, B. Inhester, T. Tadesse, A critical assessment of nonlinear forcefree field modeling of the solar corona for active region 10953. Astrophys. J. 696, 1780-1791 (2009). doi:10.1088/0004-637X/696/2/1780

C. Downs, J.A. Linker, Z. Mikić, P. Riley, C.J. Schrijver, P. Saint-Hilaire, Probing the solar magnetic field with a sun-grazing comet. Science 340, 1196-1199 (2013). doi:10.1126/science.1236550

G.A. Gary, Plasma beta above a solar active region: rethinking the paradigm. Sol. Phys. 203, 71-86 (2001). doi:10.1023/A:1012722021820 
S.E. Gibson, F. Bagenal, Large-scale magnetic field and density distribution in the solar minimum corona. J. Geophys. Res. 100, 19,865-19,880 (1995). doi:10.1029/95JA01905

S.E. Gibson, F. Bagenal, B.C. Low, Current sheets in the solar minimum corona. J. Geophys. Res. 101, 4813-4824 (1996). doi:10.1029/95JA03477

S. Gosain, A.A. Pevtsov, Resolving azimuth ambiguity using vertical nature of solar quiet-sun magnetic fields. Sol. Phys. 283, 195-205 (2013). doi:10.1007/s11207-012-0135-1, arXiv:1210.6691

H. Grad, H. Rubin, in Proc. 2nd Intern. Conf. on Peaceful Uses of Atomic Energy, vol. 31, pp. 190-+ (1958)

J.T. Hoeksema, Structure and evolution of the large scale solar and heliospheric magnetic fields. Ph.D. thesis, Stanford Univ., CA (1984)

R. Howard, Magnetic field of the Sun (observational). Annu. Rev. Astron. Astrophys. 5, 1 (1967). doi:10.1146/annurev.aa.05.090167.000245

J. Jing, C. Tan, Y. Yuan, B. Wang, T. Wiegelmann, Y. Xu, H. Wang, Free magnetic energy and flare productivity of active regions. Astrophys. J. 713, 440-449 (2010). doi:10.1088/0004-637X/713/1/440

H.P. Jones, Recent studies of magnetic canopies. Aust. J. Phys. 38, 919-928 (1985)

M. Kramar, B. Inhester, S.K. Solanki, Vector tomography for the coronal magnetic field. I. Longitudinal Zeeman effect measurements. Astron. Astrophys. 456, 665-673 (2006). doi:10.1051/0004-6361:20064865

M. Kramar, B. Inhester, H. Lin, J. Davila, Vector tomography for the coronal magnetic field. II. Hanle effect measurements. Astrophys. J. 775, 25 (2013). doi:10.1088/0004-637X/775/1/25

H. Lin, J.R. Kuhn, R. Coulter, Coronal magnetic field measurements. Astrophys. J. Lett. 613, L177-L180 (2004). doi:10.1086/425217

R. Lionello, Z. Mikić, J.A. Linker, Magnetohydrodynamics of solar coronal plasmas in cylindrical geometry. J. Comput. Phys. 140, 172 (1998)

R. Lionello, M. Velli, C. Downs, J.A. Linker, Z. Mikić, A. Verdini, Validating a time-dependent turbulencedriven model of the solar wind. Astrophys. J. 784, 120 (2014). doi:10.1088/0004-637X/784/2/120, arXiv: 1402.4188

Y. Liu, H. Lin, Observational test of coronal magnetic field models. I. Comparison with potential field model. Astrophys. J. 680, 1496-1507 (2008). doi:10.1086/588645, arXiv:0710.3223

W.C. Livingston, J. Harvey, A.K. Pierce, D. Schrage, B. Gillespie, J. Simmons, C. Slaughter, Kitt peak 60-cm vacuum telescope. Appl. Opt. 15, 33-39 (1976). doi:10.1364/AO.15.000033

B.C. Low, Three-dimensional structures of magnetostatic atmospheres. III-A general formulation. Astrophys. J. 370, 427-434 (1991). doi:10.1086/169829

A. Malanushenko, C.J. Schrijver, M.L. DeRosa, M.S. Wheatland, Using coronal loops to reconstruct the magnetic field of an active region before and after a major flare. Astrophys. J. 783, 102 (2014). doi:10.1088/0004-637X/783/2/102, arXiv:1312.5389

E. Marsch, Kinetic physics of the solar corona and solar wind. Living Rev. Sol. Phys. 3, 1 (2006). doi:10.12942/lrsp-2006-1

T.R. Metcalf, M.L. De Rosa, C.J. Schrijver, G. Barnes, A.A. van Ballegooijen, T. Wiegelmann, M.S. Wheatland, G. Valori, J.M. McTtiernan, Nonlinear force-free modeling of coronal magnetic fields. II. Modeling a filament arcade and simulated chromospheric and photospheric vector fields. Sol. Phys. 247, 269-299 (2008). doi:10.1007/s11207-007-9110-7

Z. Mikić, J.A. Linker, Disruption of coronal magnetic field arcades. Astrophys. J. 430, 898 (1994)

T. Neukirch, On self-consistent three-dimensional analytic solutions of the magnetohydrostatic equations. Astron. Astrophys. 301, 628 (1995)

G.J.D. Petrie, Three-dimensional Equilibrium Solutions to the Magnetohydrodynamic Equations and their Application to Solar Coronal Structures. Ph.D. thesis, Department of Mathematics and Statistics, University of St. Andrews, North Haugh, St Andrews KY16 9SS (2000)

G.J.D. Petrie, T. Neukirch, The Green's function method for a special class of linear three-dimensional magnetohydrostatic equilibria. Astron. Astrophys. 356, 735-746 (2000)

G.J.D. Petrie, I. Patrikeeva, A comparative study of magnetic fields in the solar photosphere and chromosphere at equatorial and polar latitudes. Astrophys. J. 699, 871-884 (2009). doi:10.1088/0004637X/699/1/871, arXiv:1010.6041

G.J.D. Petrie, A. Canou, T. Amari, Nonlinear force-free and potential-field models of active-region and global coronal fields during the whole heliosphere interval. Sol. Phys. 274, 163-194 (2011). doi:10.1007/s11207-010-9687-0, arXiv:1010.6283

G.J.D. Petrie, K. Petrovay, K. Schatten, Solar polar fields and the 22-year activity cycle: observations and models. Space Sci. Rev. 186, 325-357 (2014). doi:10.1007/s11214-014-0064-4

L.A. Rachmeler, S.E. Gibson, J.B. Dove, C.R. DeVore, Y. Fan, Polarimetric properties of flux ropes and sheared arcades in coronal prominence cavities. Sol. Phys. 288, 617-636 (2013). doi:10.1007/ s11207-013-0325-5, arXiv:1304.7594

P. Riley, J.G. Luhmann, Interplanetary signatures of unipolar streamers and the origin of the slow solar wind. Sol. Phys. 277, 355-373 (2012). doi:10.1007/s11207-011-9909-0 
P. Riley, J.A. Linker, Z. Mikić, An empirically-driven global MHD model of the corona and inner heliosphere. J. Geophys. Res. 106, 15,889 (2001). doi:10.1029/2000JA000121

P. Riley, J.A. Linker, Z. Mikić, R. Lionello, S.A. Ledvina, J.G. Luhmann, A comparison between global solar magnetohydrodynamic and potential field source surface model results. Astrophys. J. 653, 1510 (2006). doi:10.1086/508565

P. Riley, J.A. Linker, R. Lionello, Z. Mikic, Corotating interaction regions during the recent solar minimum: The power and limitations of global MHD modeling. J. Atmos. Sol.-Terr. Phys. 83, 1-10 (2012a). doi:10.1016/j.jastp.2011.12.013

P. Riley, R. Lionello, J.A. Linker, Z. Mikic, J. Luhmann, J. Wijaya, Global MHD modeling of the solar corona and inner heliosphere for the whole heliosphere interval. Sol. Phys. 274, 361-3775 (2012b). doi:10.1007/s11207-010-9698-X

P. Riley, J.A. Linker, C.N. Arge, On the role played by magnetic expansion factor in the prediction of solar wind speed. Accepted for publication in Space Weather (2015)

P. Ruan, T. Wiegelmann, B. Inhester, T. Neukirch, S.K. Solanki, L. Feng, A first step in reconstructing the solar corona self-consistently with a magnetohydrostatic model during solar activity minimum. Astron. Astrophys. 481, 827-834 (2008). doi:10.1051/0004-6361:20078834

V. Rušin, M. Druckmüller, P. Aniol, M. Minarovjech, M. Saniga, Z. Mikić, J.A. Linker, R. Lionello, P. Riley, V.S. Titov, Comparing eclipse observations of the 2008 August 1 solar corona with an MHD model prediction. Astron. Astrophys. 513, A45 (2010). doi:10.1051/0004-6361/200912778

K.H. Schatten, Current sheet magnetic model for the solar corona. Cosm. Electrodyn. 2, 232-245 (1971)

K.H. Schatten, J.M. Wilcox, N.F. Ness, A model of interplanetary and coronal magnetic fields. Sol. Phys. 6, 442-455 (1969). doi:10.1007/BF00146478

C.J. Schrijver, M.L. De Rosa, T.R. Metcalf, Y. Liu, J. McTiernan, S. Régnier, G. Valori, M.S. Wheatland, T. Wiegelmann, Nonlinear force-free modeling of coronal magnetic fields part I: a quantitative comparison of methods. Sol. Phys. 235, 161-190 (2006). doi:10.1007/s11207-006-0068-7

C.J. Schrijver, M.L. De Rosa, T. Metcalf, G. Barnes, B. Lites, T. Tarbell, J. McTiernan, G. Valori, T. Wiegelmann, M.S. Wheatland, T. Amari, G. Aulanier, P. Démoulin, M. Fuhrmann, K. Kusano, S. Régnier, J.K. Thalmann, Nonlinear force-free field modeling of a solar active region around the time of a major flare and coronal mass ejection. Astrophys. J. 675, 1637-1644 (2008). doi:10.1086/527413. arXiv:0712.0023

M. Schulz, E.N. Frazier, D.J. Boucher, Coronal magnetic-field model with non-spherical source surface. Sol. Phys. 60, 83-104 (1978)

X. Sun, J.T. Hoeksema, Y. Liu, T. Wiegelmann, K. Hayashi, Q. Chen, J. Thalmann, Evolution of magnetic field and energy in a major eruptive active region based on SDO/HMI observation. Astrophys. J. 748, 77 (2012). doi:10.1088/0004-637X/748/2/77, arXiv:1201.3404

L. Svalgaard, T.L.Jr. Duvall, P.H. Scherrer, The strength of the sun's polar fields. Sol. Phys. 58, 225-239 (1978). doi:10.1007/BF00157268

T. Tadesse, A.A. Pevtsov, T. Wiegelmann, P.J. MacNeice, S. Gosain, Global solar free magnetic energy and electric current density distribution of Carrington rotation 2124. Sol. Phys. 289, 4031-4045 (2014a). doi:10.1007/s11207-014-0581-z

T. Tadesse, T. Wiegelmann, S. Gosain, P. MacNeice, A.A. Pevtsov, First use of synoptic vector magnetograms for global nonlinear, force-free coronal magnetic field models. Astron. Astrophys. 562, A105 (2014b). doi:10.1051/0004-6361/201322418, arXiv:1309.5853

T. Tadesse, T. Wiegelmann, P.J. MacNeice, B. Inhester, K. Olson, A. Pevtsov, A comparison between nonlinear force-free field and potential field models using full-disk SDO/HMI magnetogram. Sol. Phys. 289, 831-845 (2014c). doi:10.1007/s11207-013-0364-y, arXiv:1212.5639

G. Tóth, B. van der Holst, Z. Huang, Obtaining potential field solutions with spherical harmonics and finite differences. Astrophys. J. 732, 102 (2011). doi:10.1088/0004-637X/732/2/102, arXiv:1104.5672

G. Valori, B. Kliem, R. Keppens, Extrapolation of a nonlinear force-free field containing a highly twisted magnetic loop. Astron. Astrophys. 433, 335-347 (2005). doi:10.1051/0004-6361:20042008

B. van der Holst, I.V. Sokolov, X. Meng, M. Jin, W.B. Manchester IV, G. Tóth, T.I. Gombosi, Alfvén Wave Solar Model (AWSoM): coronal heating. Astrophys. J. 782, 81 (2014). doi:10.1088/ 0004-637X/782/2/81, arXiv:1311.4093

Y.M. Wang, N.R. Sheeley, Solar wind speed and coronal flux-tube expansion. Astrophys. J. 355, 726 (1990). doi: $10.1086 / 168805$

Y.M. Wang, N.R.Jr. Sheeley, On potential field models of the solar corona. Astrophys. J. 392, 310-319 (1992). doi: $10.1086 / 171430$

M.S. Wheatland, K.D. Leka, Achieving self-consistent nonlinear force-free modeling of solar active regions. Astrophys. J. 728, 112 (2011). doi:10.1088/0004-637X/728/2/112, arXiv:1012.3503

M.S. Wheatland, S. Régnier, A self-consistent nonlinear force-free solution for a solar active region magnetic field. Astrophys. J. Lett. 700, L88-L91 (2009). doi:10.1088/0004-637X/700/2/L88, arXiv:0906.4414 
M.S. Wheatland, P.A. Sturrock, G. Roumeliotis, An optimization approach to reconstructing force-free fields. Astrophys. J. 540, 1150-1155 (2000). doi:10.1086/309355

T. Wiegelmann, Nonlinear force-free modeling of the solar coronal magnetic field. J. Geophys. Res. Space Phys. 113, A03S02 (2008). doi:10.1029/2007JA012432

T. Wiegelmann, B. Inhester, How to deal with measurement errors and lacking data in nonlinear force-free coronal magnetic field modelling? Astron. Astrophys. 516, A107 (2010). doi:10.1051/ 0004-6361/201014391

T. Wiegelmann, T. Sakurai, Solar force-free magnetic fields. Living Rev. Sol. Phys. 9, 5 (2012). doi:10.12942/ lrsp-2012-5, arXiv:1208.4693

T. Wiegelmann, B. Inhester, T. Sakurai, Preprocessing of vector magnetograph data for a nonlinear forcefree magnetic field reconstruction. Sol. Phys. 233, 215-232 (2006). doi:10.1007/s11207-006-2092-z, arXiv:astro-ph/0612641

T. Wiegelmann, T. Neukirch, P. Ruan, B. Inhester, Optimization approach for the computation of magnetohydrostatic coronal equilibria in spherical geometry. Astron. Astrophys. 475, 701-706 (2007). doi:10.1051/0004-6361:20078244, arXiv:0801.2916

T. Wiegelmann, J.K. Thalmann, C.J. Schrijver, M.L. De Rosa, T.R. Metcalf, Can we improve the preprocessing of photospheric vector magnetograms by the inclusion of chromospheric observations? Sol. Phys. 247, 249-267 (2008). doi:10.1007/s11207-008-9130-y, arXiv:0801.2707

T. Wiegelmann, J.K. Thalmann, B. Inhester, T. Tadesse, X. Sun, J.T. Hoeksema, How should one optimize nonlinear force-free coronal magnetic field extrapolations from SDO/HMI vector magnetograms? Sol. Phys. 281, 37-51 (2012). doi:10.1007/s11207-012-9966-z, arXiv:1202.3601

T. Wiegelmann, J.K. Thalmann, S.K. Solanki, The magnetic field in the solar atmosphere. Astron. Astrophys. Rev. 22, 78 (2014). doi:10.1007/s00159-014-0078-7, arXiv:1410.4214

A.R. Yeates, D.H. Mackay, Chirality of high-latitude filaments over Solar Cycle 23. Astrophys. J. Lett. 753, L34 (2012). doi:10.1088/2041-8205/753/2/L34, arXiv:1206.2327

X. Zhao, J.T. Hoeksema, A coronal magnetic field model with horizontal volume and sheet currents. Sol. Phys. 151, 91-105 (1994). doi:10.1007/BF00654084

X.P. Zhao, J.T. Hoeksema, P.H. Scherrer, Modeling the 1994 April 14 polar crown SXR arcade using three-dimensional magnetohydrostatic equilibrium solutions. Astrophys. J. 538, 932-939 (2000). doi:10.1086/309166 